\title{
1 Subjective understanding of actions and emotions involves the 2 interplay of the semantic and action observation networks in
}

3 the brain

4 Minye Zhan ${ }^{12}$, Rainer Goebel ${ }^{13}$, and Beatrice de Gelder ${ }^{14 *}$

$5{ }^{1}$ Faculty of Psychology and Neuroscience, Department of Cognitive Neurosciences, Maastricht University,

6 6229EV Maastricht, The Netherlands

$7 \quad{ }^{2}$ U992 (Cognitive neuroimaging unit), NeuroSpin, INSERM-CEA, 91191 Gif sur Yvette, France

$8{ }^{3}$ Department of Neuroimaging and Neuromodeling, Netherlands Institute for Neuroscience, Institute of

9 the Royal Netherlands Academy of Arts and Sciences, 1105 BA, Amsterdam, The Netherlands

$10 \quad{ }^{4}$ Department of Computer Science, University College London, WC1E 6BT, UK

$12 *$ Corresponding author and lead contact: b.degelder@maastrichtuniversity.nl

\section{Summary}

14 How we subjectively generate an understanding of other people's bodily actions and emotions is

15 not well understood. In this 7T fMRI study, we examined the representational geometry of

16 bodily action- and emotion-understanding by mapping individual subjective reports with word

17 embeddings, besides using conventional univariate/multivariate analyses with predefined

18 categories. Dimensionality reduction revealed that the representations for perceived action and

19 emotion were high dimensional, each correlated to but were not reducible to the predefined

20 action and emotion categories. With searchlight representational similarity analysis, we found

21 the left middle superior temporal sulcus and left dorsal premotor cortex corresponded to the

22 subjective action and emotion representations. Furthermore using task-residual functional

23 connectivity and hierarchical clustering, we found that areas in the action observation network

24 and the semantic/default-mode network were functionally connected to these two seed regions

25 and showed similar representations. Our study provides direct evidence that both networks

26 were concurrently involved in subjective action and emotion understanding.

27 Keywords: body, action, emotion, subjective report, 7T fMRI, representational similarity analysis 
bioRxiv preprint doi: https://doi.org/10.1101/2021.04.15.439961; this version posted April 15, 2021. The copyright holder for this preprint (which was not certified by peer review) is the author/funder, who has granted bioRxiv a license to display the preprint in perpetuity. It is made available under aCC-BY-NC-ND 4.0 International license.

\section{Graphical abstract}

40 stimuli, 10 action categories

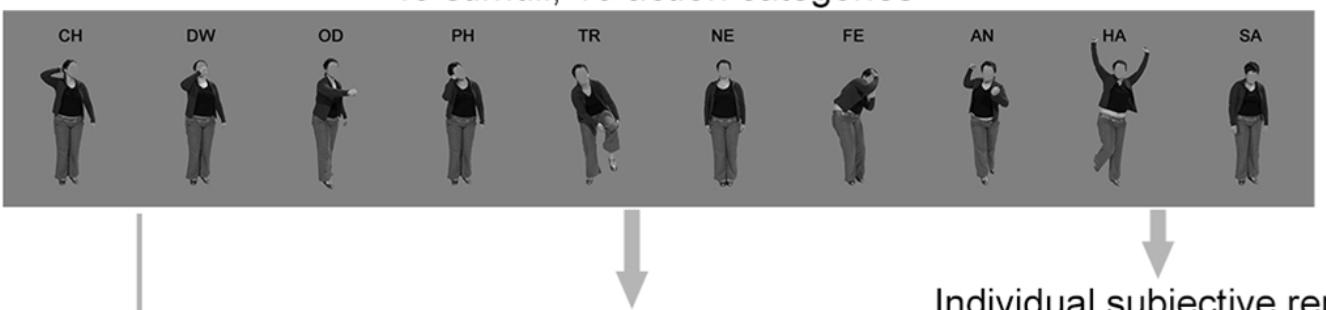

Individual subjective reports Individual 7T fMRI data

Predefined actions, emotions

$1.2 \mathrm{~mm}$ isotropic, $\mathrm{TR}=2 \mathrm{~s}$

Deconf word vectors
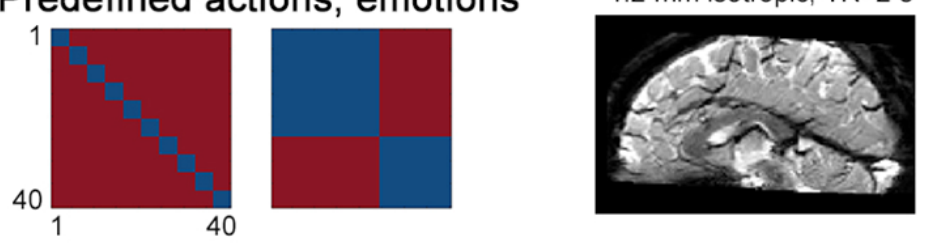

Perceived actions, emotions

The perceived action network and perceived emotion network overlapped with the DMN/semantic network
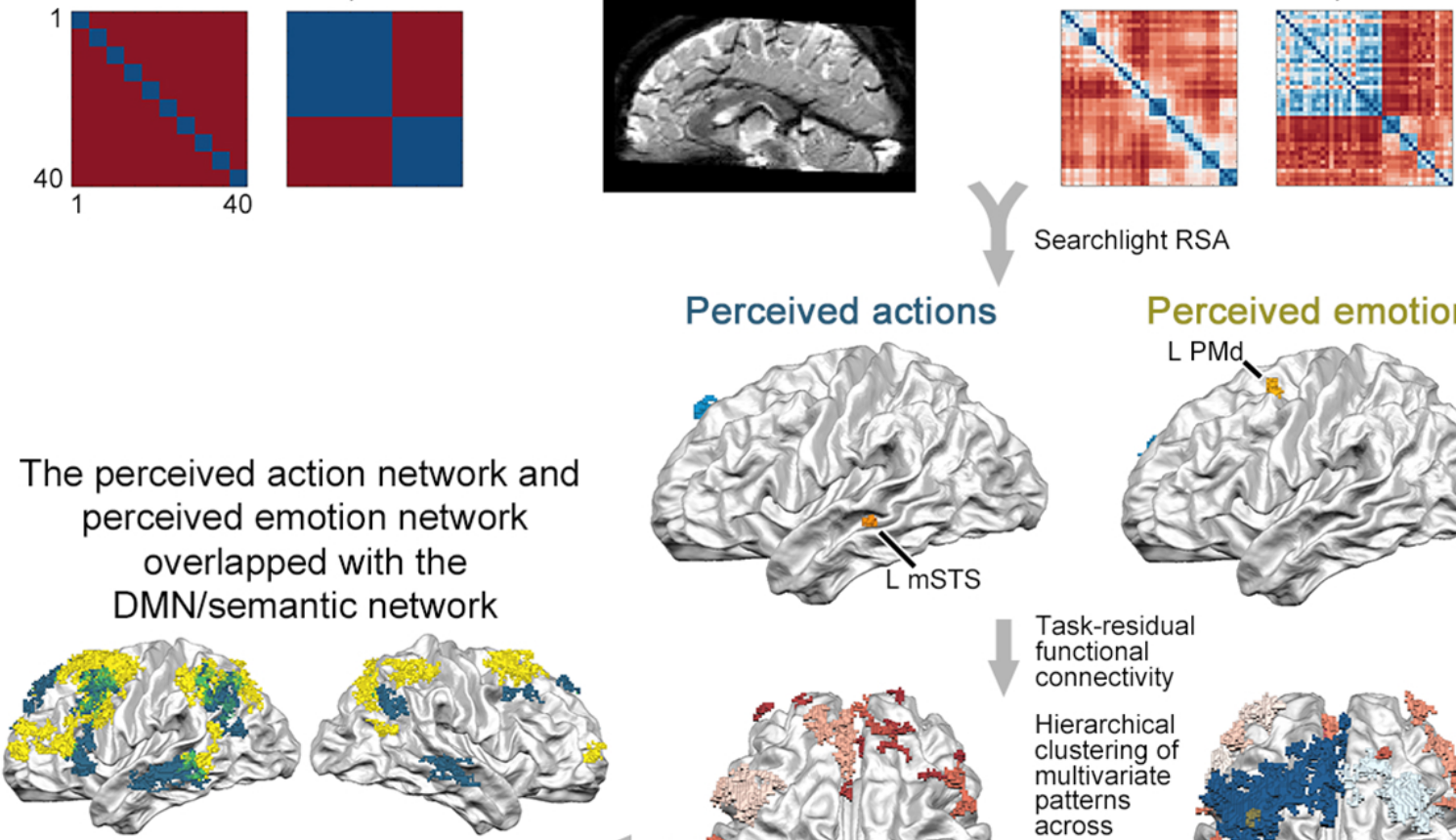

Searchlight RSA
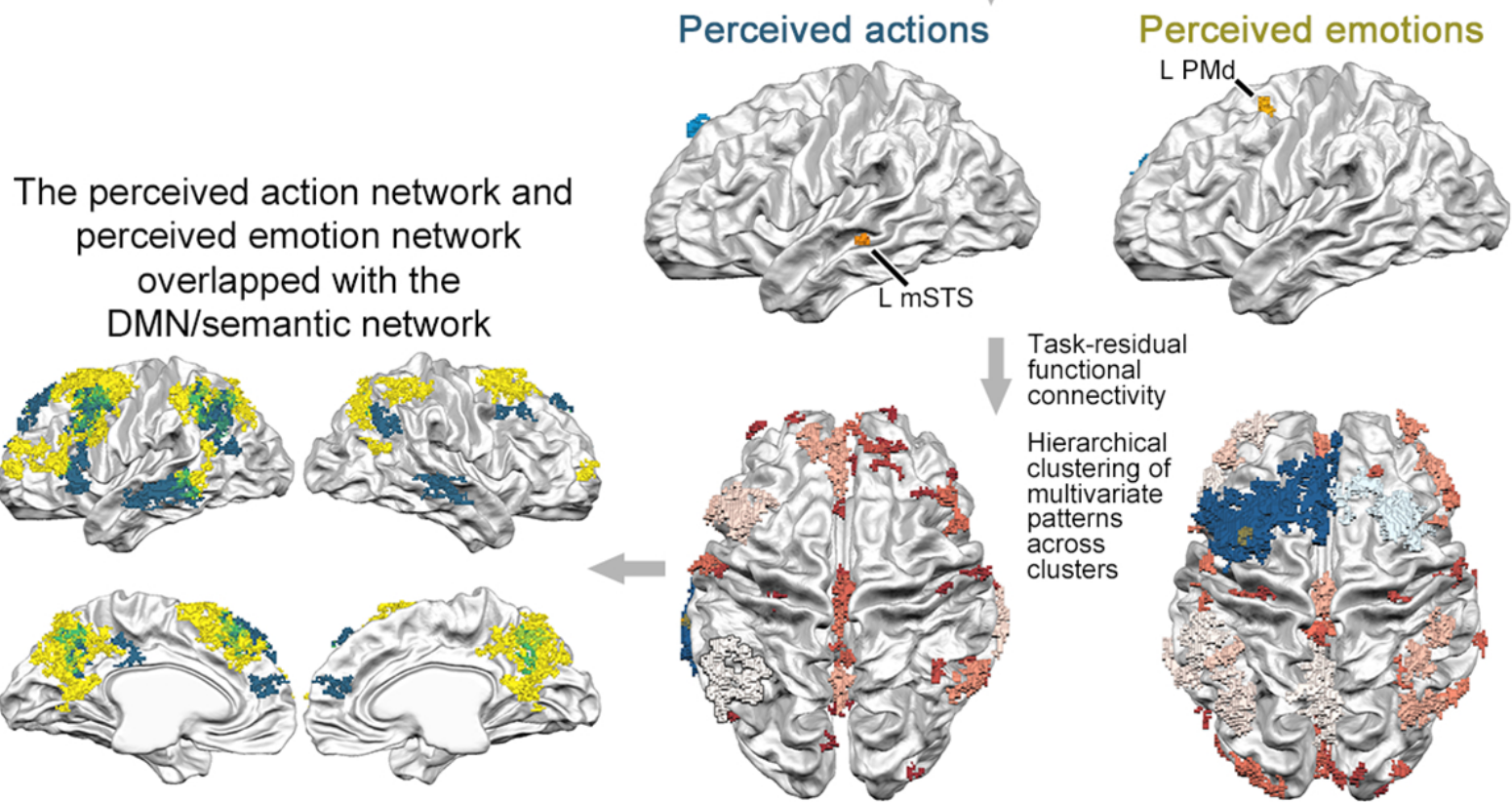

Task-residual

functional

connectivity

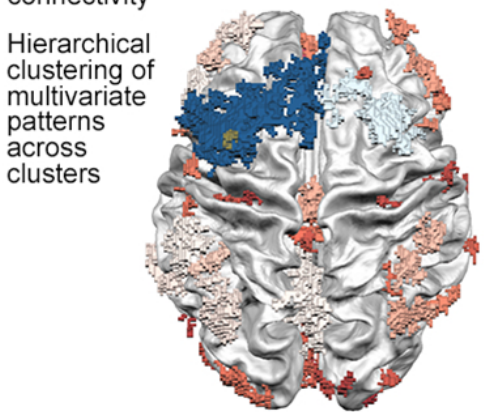




\section{Introduction}

32 Imagine you are walking into a large conference hall and you see a person waving in your direction from far away. Within hundreds of milliseconds, this triggers a cascade of implicit and explicit brain processes: "Do I know this person? Is she waving towards me, perhaps to notify me something? Since I don't know her and she seems to be quite happy, is she perhaps waving towards someone close by who might be her acquaintance?" This is an example of the multifaceted information about the action, emotion, intention and identity that human bodies routinely convey, which we process effortlessly in daily life. Our brains extract and combine those various dimensions of information, enabling us to reach a personal/subjective understanding, and to act upon it.

These different dimensions of information have been the focus of specialized lines of inquiry. Univariate studies of action observation have found a fronto-parietal network (action observation network) including the intraparietal sulcus (IPS) and the dorsal and ventral premotor areas (PMd, PMv)(Caspers et al., 2010; Grafton and Hamilton, 2007; Rizzolatti et al., 2014). Studies focused on body forms found body/body parts-sensitive regions in the ventrallateral pathway, the extrastriate body area (EBA) and the fusiform body area (FBA) (Peelen and Downing, 2007), while the posterior superior temporal sulcus (pSTS) is sensitive to the biological motion of both faces and bodies (Allison et al., 2000). A study addressing the intention aspect of action (de Lange et al., 2008) reported involvement of the inferior frontal gyrus (IFG) for understanding unusual intentions, and involvement of the default mode network (DMN) (Andrews-Hanna, 2012; Buckner et al., 2008) for attending to action intentions in general.

When the body posture and movements convey emotional information, the stimuli trigger activation in areas associated with visual form, action and movement perception, in concert with more emotion-specific areas including the IFG, insula and subcortical structures (Dricu and Frühholz, 2016; de Gelder, 2006; de Gelder et al., 2004; Kober et al., 2008; Lindquist et al., 2012; Molenberghs et al., 2012; Sinke et al., 2010). DMN areas including the temporo-parietal junction (TPJ), precuneus, dorsal/ventral medial prefrontal cortex (dmPFC, vmPFC) (Amodio and Frith, 2006; Saxe et al., 2006), are involved in emotion and valence processing (Chikazoe et al., 2014; Peelen et al., 2010; Skerry and Saxe, 2015), as revealed with multivariate methods such as representational similarity analysis (RSA) (Kriegeskorte et al., 2008; Nili et al., 2014). However univariate activation of DMN areas in action- and emotion-related studies had been scarce, the DMN was considered as a separate system that does not interact with the action observation network (Van Overwalle and Baetens, 2009). categories, and often used an explicit action or emotion recognition/categorization task, serving as a proxy for subjective action and emotion understanding. This approach also conforms to the traditional view of a few basic emotions (e.g. Ekman, 1999), assuming that (1) there exist a few discrete categories of actions and emotions that participants are routinely able to recognize; (2) there is high inter-individual similarity in recognizing and identifying these categories; (3) there 
is a stable brain-behavior mapping between action and emotion categories and brain processes

71 across participants.

72 Under these assumptions, individual variability in perceiving the categories is typically treated as noise. However, individual variability is prevalent in multiple functions from perception to cognition, and some individual variability can be linked to the size or function of specific brain areas (for reviews see Kanai and Rees, 2011; Seghier and Price, 2018). Specifically concerning action and emotion perception, the first two assumptions of discrete categories and high interindividual similarity may not hold either. Different observers may well reach a different understanding of the action/emotion, depending on factors such as the expressiveness of each expression (as could often be observed in validation studies for expression databases, e.g. Goeleven et al., 2008; Langner et al., 2010), the personality of the viewer (e.g. Van den Stock et al., 2015), and various contexts (for a review see Aviezer et al., 2008; for examples see Kret and de Gelder, 2010; Righart and de Gelder, 2006). However, the third assumption may still hold. Different individual behavior may still map to the same neural underpinning, as one fMRI study found individualized and unique dissimilarity ratings for objects were still represented in the brain activity patterns in participants' inferior temporal cortices (Charest et al., 2014).

The tendency to treat individual variability as noise was also partially related to the difficulty to objectively quantify individual variability across participants, especially for the case of verbal reports. With the advancement of deep neural networks and natural language processing techniques, word embeddings (see Boleda, 2020 for a review) are now used to describe semantic concepts in an objective and quantitative way, and recent studies started linking them to brain activity (Hebart et al., 2019; Zhang et al., 2020).

Recently, researchers argued that studying subjective experiences is important for understanding the high-level cognitions such as emotion, language, and music (Hartley and Poeppel, 2020; LeDoux and Hofmann, 2018). The presence of subjective variability in emotional perception and production is also acknowledged in the recent literature (for a review for facial expressions, see Barrett et al., 2019; for a review for more general emotional stimuli, see Cowen and Keltner, 2021). In response to these challenges, new studies utilized very large stimulus sets, subjective reports, and the associated semantic space (Cowen and Keltner, 2017, 2020, 2021; Cowen et al., 2019). Results indicated that for the subjective experiences elicited by facial, vocal, musical emotional expressions, over 20 emotional categories could be observed in the subjective reports for some modalities. There were no discrete category boundaries, and the semantic space of these categories was also high-dimensional. The brain-behavior mappings of such rich emotion representations seen in subjective reports were largely uncharted, independent from the pre-defined basic categories.

In this high-resolution 7T fMRI study (1.2 mm isotropic resolution, voxel volume $=1.728 \mathrm{~mm}^{3}$ ) with RSA (Kriegeskorte et al., 2008; Nili et al., 2014), our goal was to address both sides, the 
of 10 predefined bodily action categories ( 6 neutral, 4 emotional categories; each participant viewed 40 stimuli). Immediately following the scanning session, participants provided free reports of the subjectively perceived action and emotion for each stimulus.

We first examined the representational geometry of the subjective behavioral reports by principal component analysis (PCA) and RSA, after mapping all subjective report entries into a common vector space using Deconf word embeddings (Pilehvar and Collier, 2016). Then we examined the neural representations for predefined categories with RSA searchlight and RSA regression. There we estimated the body joint positions using the OpenPose library (Cao et al., 2017), and computed low- and mid-level visual features. Lastly, we examined the relation between subjective reports and brain activity. We searched for the subjective representations in individual participant data by RSA searchlight, and examined their putative direct upstream/downstream areas by task-residual functional connectivity and hierarchical clustering. We found neural representations for perceived action and perceived emotion, and the two analyses with predefined categories and with the subjective reports converged as they both indicated joint involvement of the action observation network and the DMN/semantic network.

\section{Results}

\section{RDM construction}

In the scanning session, participants passively viewed 40 gray-scale images from one of two balanced stimuli sets (Fig. 1A. See Fig. S1 for the complete sets of stimuli). We used a slow event-related design, where each image ( $2.60 \times 4.26$ degrees) was presented for $500 \mathrm{ms,}$ followed by an inter-stimulus interval of either 7.5, 9.5 or $11.5 \mathrm{~s}$. Each image was presented 12 times.

In the behavioral session directly after the fMRI scan, for each stimulus image, participants reported their subjective understanding of the action and emotion by typing a short description, and rated the implied motion and valence on a scale of 1 to 7 (See Table S1 for the exact questions, and examples of participants' free reports).

Individual reports were analyzed by mapping all response entries to the same high-dimensional space using the pre-trained Deconf word embeddings (Pilehvar and Collier, 2016), which combined the word2vec word embeddings and the WordNet database (see Methods for details). Specifically, we lemmatized all the verbs, nouns, adjectives, adverbs typed in by the participants (e.g. fighting/fights $\rightarrow$ fight), and selected the corresponding meaning for each word in WordNet 3.1 (https://wordnet.princeton.edu/). We then retrieved the corresponding 300dimensional vectors from Deconf embeddings, averaged the word vectors in each response entry, and computed RDMs for perceived action and perceived emotion (cosine distance) for each individual participant, see Fig. 1Bab and 1Cab. 
Implied motion and valence ratings were one-dimensional attributes provided by individual participants, which were related respectively to the action and emotion aspects of each stimulus. These RDMs were computed directly from the individual behavioral ratings (Euclidean distance, Fig. 1Bcd, Ccd).

For predefined categories (10 action categories, non-emotional/emotional actions), we followed the RSA and perceptual categorization literature and constructed model RDMs (Euclidean distance, Fig. 1B, first column), assuming that the stimuli representations were similar within categories, but were different across categories between stimuli (e.g. Freedman et al., 2001).

We examined the correlations of the subjective report and the rating RDMs with the two predefined RDMs. Throughout the whole study, correlations between RDMs were computed with Spearman correlation, and submitted to a one-sample t test against 0 (two-tailed) at the group level, after Fisher's Z transformation.

\section{Perceived categories largely corresponded to the predefined ones, but with considerable individual variability}

All four subjective report and rating RDMs were significantly correlated to the predefined ones (one-sample t test against 0 ; coefficient of variation, $\mathrm{CV}$, showing inter-individual variability), perceived action to predefined action RDMs: mean rho $=0.324, p=8.55 \times 10^{-8}, C V=20.4 \%$; perceived emotion to predefined emotion RDMs: mean rho $=0.491, p=0.000164, C V=51.2 \%$; rated implied motion to the predefined action RDMs: mean rho $=0.166, p=0.000541, C V=60.4 \%$; rated valence to the predefined emotion $\mathrm{RDM}$ : mean $\mathrm{rho}=0.496, p=5.91 \times 10^{-7}, \mathrm{CV}=25.5 \%$. This indicates that the individual subjective understanding largely corresponded to the predefined categories, especially for emotions, although there was considerable individual variability (Fig. 1C, D).

We then examined the individual variability, to assess whether it is suitable to use groupaveraged RDMs to perform further analyses in individual participants. We first obtained a groupaveraged RDM from all 10 individual RDMs, then computed its Spearman correlation to each individual RDM (one-sample t test against $0 ; \mathrm{CV}$ ). When inter-individual consistency is high, the group-averaged RDM should have high correlation to each individual RDM, and a low CV. For valence, the group-averaged RDM was very consistent with individual RDMs: mean rho $=0.900$, $p=6.91 \times 10^{-8}, C V=19.9 \%$. However, the other 3 group-averaged behavioral RDMs were less consistent with individual RDMs, again showing considerable individual variability: perceived action RDM: mean rho $=0.517, \mathrm{p}=4.74 \times 10^{-6}, \mathrm{CV}=32.7 \%$; perceived emotion RDM: mean rho $=0.565, p=6.02 \times 10^{-5}, C V=44.9 \%$; rated implied motion RDM: mean rho $=0.621, p=8.71 \times 10^{-5}$, $\mathrm{CV}=47.1 \%$. With these moderate correlation coefficients, indicating less tight brain-behavior links for individual participants, using group-averaged RDMs in subsequent analyses may be less suitable to capture the individualized neural substrates. It was also the case for the pre-defined categories. 
On the other hand, analyses based on the individual subjective responses may better capture the individual neural processes, because the neural activity and patterns generating these individualized subjective reports (behavioral outputs) may still map to brain locations in a consistent and meaningful way. If the neural processes found this way are robust and consistent enough, individual-level replication can be achieved (Smith and Little, 2018). Adopting this logic, our subsequent analyses looked at the mapping of subjective reports to brain activity, and accordingly focuses on individual-level data and results.

The perceived action and emotion representations are highdimensional

For investigating perceived action and perceived emotion, we examined the high dimensional space of all responses using principal component analysis (PCA), since all 400 subjective responses were in the same high-dimensional space, each represented by a 300-dimensional vector.

The first few principal components (PCS) for the perceived action and perceived emotion representations captured a large amount of variance (First 10 PCs: $86.17 \%$ and $89.63 \%$ variance; first 50 PCs: $97.76 \%$ and $99.18 \%$ variances, Fig. $1 F, G$, analysis performed on $1^{\text {st }}$ to $50^{\text {th }}$ PC for both representations).

The perceived action representation RDM showed considerable individual variability when compared to the 10-action-category model (Fig. 1Ea, Ga). The first and biggest few components did not show clear separation of action categories. Instead, individual variability was more prominent (Fig. 1H), while it remains elusive what accounts for the majority of the variance in PC 1. Only the later and much smaller PCs were correlated more to the 10-action-category model $\left(7^{\text {th }}, 9^{\text {th }}, 10^{\text {th }}\right.$ PCs $)$, the non-emotion/emotion model $\left(7^{\text {th }}, 22^{\text {nd }}, 6^{\text {th }}\right.$ PCs $)$, and the implied motion ratings $\left(6^{\text {th }}, 10^{\text {th }}, 18^{\text {th }} \mathrm{PCs}\right)$, and also showed relatively clear category separations (Fig. $\mathbf{1 H}, \mathbf{I})$. The presence of smaller PCs correlated to the action categories, emotions and implied motion ratings indicates that the perceived action representation is rich and high-dimensional. It potentially supports extracting the relevant categorical and continuous information, but it is not equivalent and not reducible to one of the predefined category structures.

The perceived emotion representation was lower-dimensional than the perceived action representation (79 versus 174 independent PCs), and showed higher inter-individual consistency (Fig. 1Eb, Gb, much lower correlation value to the individual subject model in Fig. 1H). The first few PCs showed a moderate correlation to the non-emotion/emotion model and valence ratings, and even to the 10 -action-category model (Fig. $\mathbf{1 H}, \mathbf{J})$. Interestingly, visual inspection of the RDMs showed that the perceived emotion representation contained the separation of nonemotion and emotion categories (Fig. 1Eb, PC1 and 2 in Fig. 1Gb), but also contained finergrained separation for individual emotions. These were captured by the $3^{\text {rd }}, 4^{\text {th }}$ and $6^{\text {th }}$ PCs, corresponding to happy, fearful and sad (Fig. 1Gb, see the locations of the prominent white/red bars across all conditions, showing similarity within the category and dissimilarity to the other 9 categories). In the $3^{\text {rd }}$ and $4^{\text {th }}$ PC, the angry emotion could further be separated. This indicates 
221 that the perceived emotion representation is also a high-dimensional one; brain areas

222 containing such representations could potentially support both the non-emotion/emotion

223 categorization, and categorization of each individual emotions.
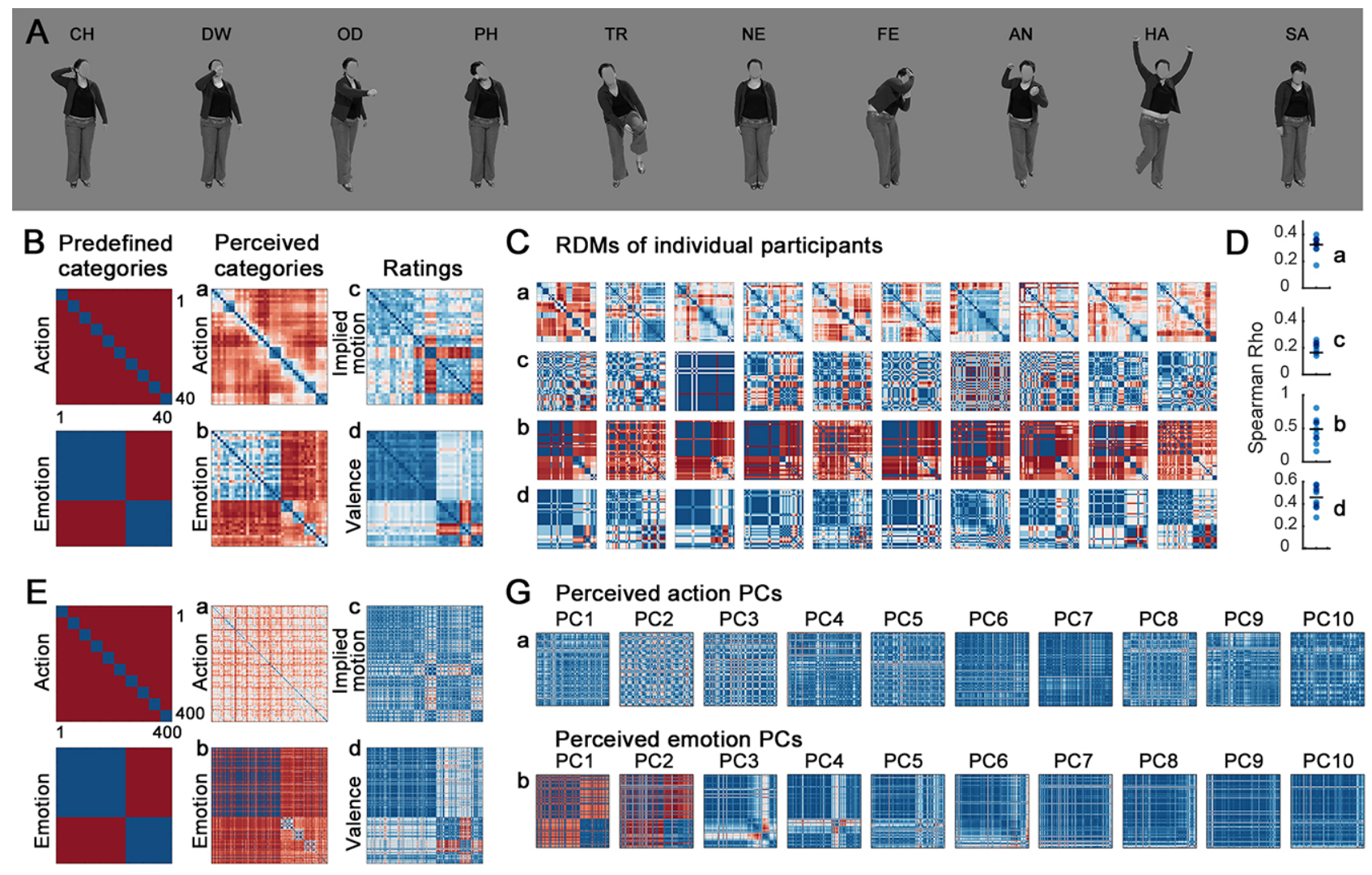

G Perceived action PCs
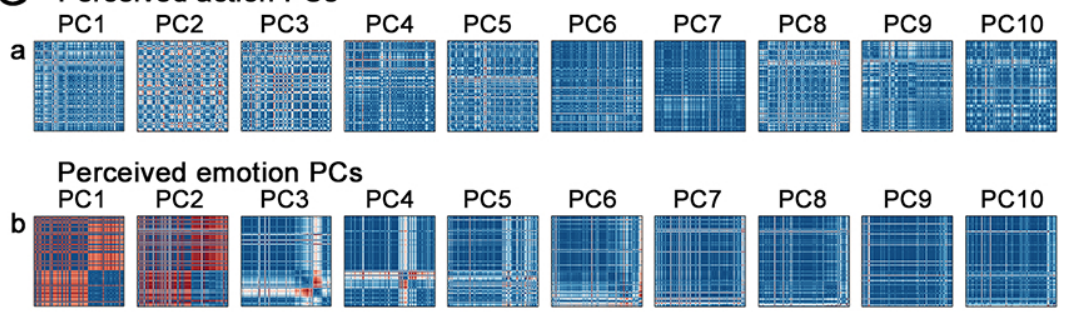

F Perceived action Perceived emotion
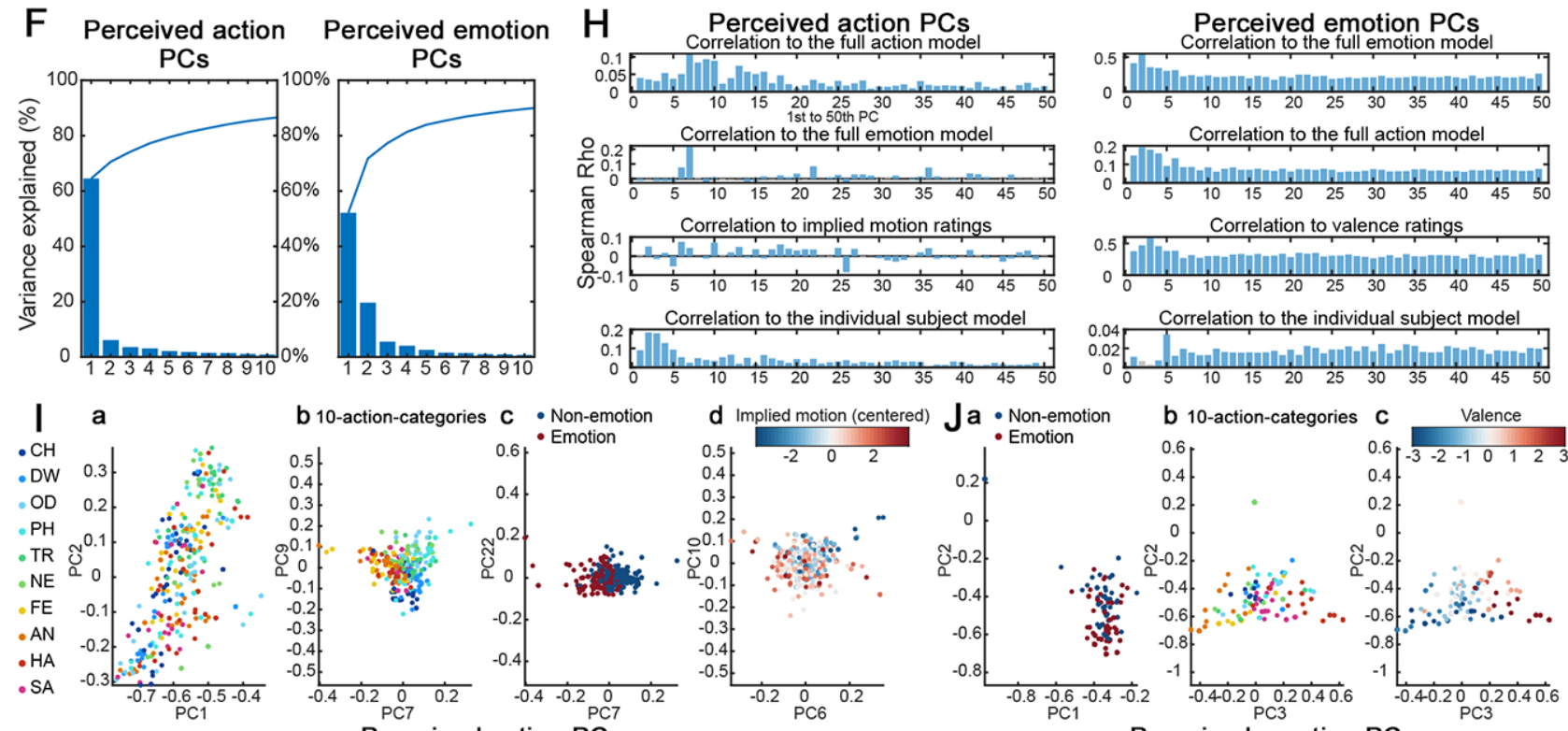

Perceived emotion PCs

225 Figure 1. The representational geometries of subjective reports were high-dimensional, and

226 showed considerable individual variability. 
A. Stimuli examples of 10 action categories, performed by one of 8 actors. Category abbreviations: $\mathrm{CH}$ : combing hair; DW: drinking water; OD: opening door; $\mathrm{PH}$ : phone; TR: putting on trousers; NE: neutral standing still; FE: fearful; AN: angry; HA: happy; SA: sad. participants ( 40 by 40 matrices, correlation distances $1-r$, all diagonals were 0 , sorted by the 10 action categories). The group-averaged RDMs showed correspondence to the predefined RDMs. a. perceived action; b. perceived emotion; c. rated implied motion; $\mathbf{d}$. rated valence. In B, C, E, G: blue color: similar; red color: dissimilar. For all RDMs, the color ranges were scaled automatically by the minimal/maximal values within each RDM. C. RDMs for individual participants showed considerable individual variability. RDM types a to d correspond to a to $\mathbf{d}$ in $\mathbf{B}$. Each column corresponds to one participant. The left 5 participants viewed stimulus set $A$, the other 5 viewed set $B$.

D. Correlation coefficients for individual participants were not high. a. perceived action correlated to the 10- action-category model; $\mathbf{b}$. perceived emotion correlated to the nonemotion/emotion model; c. implied motion ratings correlated to the 10-action-category model; d. valence ratings correlated to the non-emotion/emotion model. Black bars: group average. E to J. PCA results of all 400 behavioral report items for perceived action and perceived emotion. The smaller PCs of the perceived action and the first PCs of the perceived emotion showed correspondence to the predefined RDMs.

E. Same to B, but for all 400 behavioral report items across participants ( 400 by 400 matrices).

F. The first 10 principal components (PCs) explained a large amount of variance. Curves: cumulative explained variance (\%).

G. RDMs for scores of the first 10 PCs (Euclidean distance), for perceived action (a) and perceived emotion (b). $\mathrm{H}$. RDMs the first $50 \mathrm{PCs}$, correlated to the predefined action, emotion models, behavioral rating models, and to the individual subject model. The first PCs of the perceived action correlated more to the individual subject model. Gray bars: non-significant correlation values (FDR q>0.05). I. Perceived action report items, plotted against the first two PCs, and against the two PCs with highest correlation coefficients to each of the models. with highest correlation coefficients to each of the models. Fewer dots were shown on the plots,

\section{Univariate results were consistent with the literature}

261 For the fMRI data, as a sanity check, we first performed conventional group-level univariate

262 analysis with data smoothing, for the 10 action categories (input for the participants), and

263 parametric modulations for implied motion and valence. The 7T data was very robust: the

264 activation location and time course profiles at 7T were similar to 3T ones, but showed higher \%265 signal change (See Figure S2). 
The 10-category one-way ANOVA showed differences across action categories in bilateral EBA, right FBA, right lateral occipital cortex (LOC) and right mIPS (Fig. 2A). Similarly, the parametric modulation of implied motion showed higher activity for actions rated with higher implied motion, in bilateral EBA, right mIPS, left pIPS, right rostral cingulate zone (RCZ), where the former 3 overlapped with the ANOVA clusters (Fig. 2B).

271 When contrasting emotional and non-emotional categories (excluding the standing-still

272 condition), the left IFG and left middle temporal gyrus (MTG) showed higher activity for

273 emotional categories, while early visual areas (EVC) and right precentral gyrus showed higher

274 activity for non-emotional categories (Fig. 2C). The activation map was very similar when

275 including the standing-still condition. These univariate results were consistent with previous

276 findings (Dricu and Frühholz, 2016; de Gelder et al., 2004; Kober et al., 2008; Molenberghs et al.,

277 2012; Sinke et al., 2010). However, for the parametric modulation of valence, we only observed

278 clusters modulated by positive valence, but not for ratings of negative valence (negative

279 modulation). These clusters were found outside the frontal lobe, in bilateral early visual cortices

280 (including bilateral V3a and right calcarine sulcus/cuneus), right posterior collateral sulcus, right

281 supramarginal gyrus, left precentral gyrus/central sulcus (Fig. 2D).

282 We cannot exclude that some of these may be false negatives due to the exacerbated inter283 individual variability at 7T, where activation clusters were small, and were constrained to the 284 gray matter even after spatial smoothing. This effect could be observed in the functional 285 localizer data: despite that we found robust EBA, FBA, FFA clusters in all individual participants 286 (data smoothed $3 \mathrm{~mm}$ FWHM, contrasts: bodies>faces, houses, tools, words; faces>bodies, 287 houses, tools, words), and the activation sites were reliable in one participant between 3T and 2887 7T scans on different days (Figure S2), the across-participant overlapping of these clusters were 289 low; the group-level GLM (data smoothed $6 \mathrm{~mm}$ FWHM) only showed an R EBA/pSTS cluster for 290 bodies, L EVC and bilateral precuneus clusters for faces (Figure S3). Thus for whole-brain 291 activation and searchlight analyses, we used the initial $p$ threshold of 0.005 to balance between 292 the false positives and false negatives. 

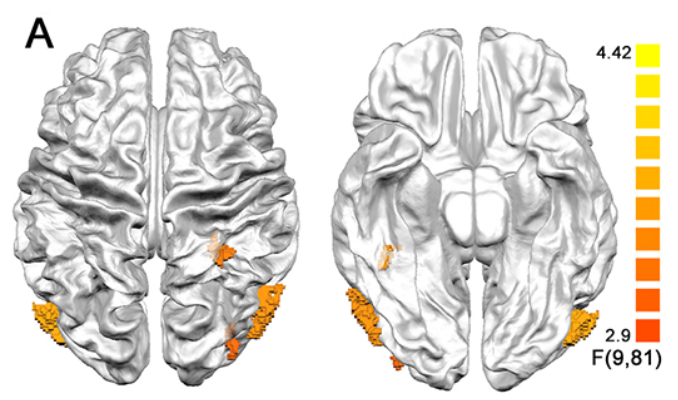

\section{C}

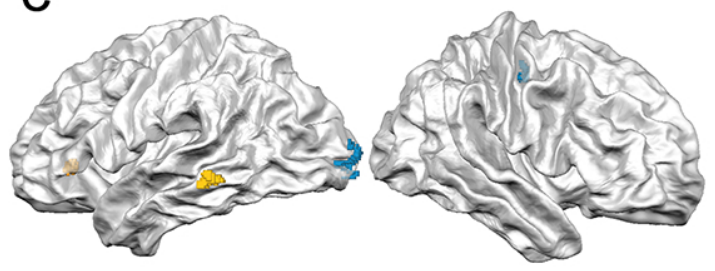

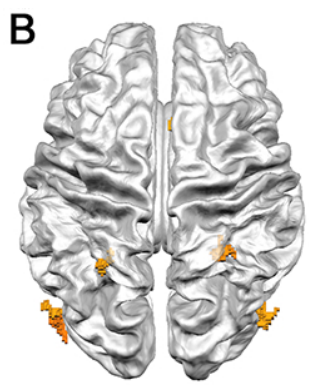

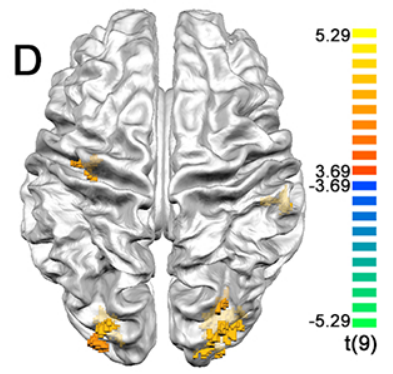

294

295

296

297

298

299

300

301

302

303

304

305

306

Figure 2. Univariate results were consistent with the literature. Data in volume space were plotted on the group-aligned surface mesh after cortex-based alignment. Functional data were smoothed at $6 \mathrm{~mm}$ FWHM, all maps were cluster-size thresholded with Monte-Carlo simulation, alpha $=0.05, n$ simulations $=5000$. We used the initial $p<0.005$ to alleviate the false negatives due to high anatomical inter-individual variability, which was clearly observed in our functional localizer data.

A. ANOVA of 10 categories. Color bar $p$ range: 0.005 to 0.0001 .

B. Parametric modulation of rated implied motion.

C. Emotional categories $>$ non-emotional categories (excluding neutral standing still).

D. Parametric modulation of rated valence. Color bar p range in B, C, D: 0.005 to 0.0005 .

Cluster size thresholds in A to D: 115, 55, 46, 56 functional voxels.

\section{Predefined non-emotion/emotion and 10-action-category representations in the brain}

Next we followed the conventional predefined-model-driven approach, basing the models on the predefined action and non-emotion/emotion categories, and performed RSA searchlight analysis for those RDMs. We constructed neural RDMs from univariate t maps of the 40 individual stimuli (no smoothing for functional data, Pearson's correlation distance), and performed RSA searchlight (radius $=5$ voxels; Spearman correlation to the predefined category RDMs, one-sampled t test against 0 for the Fisher's Z-transformed rho values at the group-level, cluster size thresholded at alpha of 0.05 , Monte-Carlo simulation $n=5000$ ). The same group-level test and cluster thresholding scheme was used for all searchlight analysis throughout the study.

For the predefined non-emotion/emotion categories, we found two areas positively correlated to the model RDM, in L central sulcus (adjacent to the cluster found in parametric modulation 
319 for valence ratings in the univariate analysis, in Fig. 2D), and R PMd/FEF (Figure 3B). Another

320 four areas showed negative correlation to the RDM, in $L$ precuneus, $L$ caudal cingulate zone,

321 bilateral medial $S F G$, and $L$ thalamus, indicating fine-grained processing for stimuli within the

322 non-emotion or emotion categories.

323 We found 29 brain areas positively correlated to the 10-action-category RDM (Figure 3A, S4,

324 Table S2, denoted "10-action-category areas" below), and no negatively-correlated areas. When

325 thresholding at $p<0.001$, eight of these areas were still present, including the $R$ cuneus, $R$

$326 \mathrm{EBA} / \mathrm{hMT}+, \mathrm{L}$ central sulcus, $\mathrm{L}$ precuneus, $\mathrm{R}$ posterior cingulate gyrus and sulcus, bilateral medial

327 superior frontal gyrus, anterior RCZ. Some of these 10-action-category areas consistently

328 showed univariate activation in body or action perception, including R EBA/hMT+, L pIPS and L

329 RCZ; but several areas did not show above-baseline univariate activation at the group level,

330 including $L$ central sulcus, $R$ anterior SFS, R cerebellum, L vmPFC and bilateral medial SFG (Figure

331 S5, Table S3).

332 We next examined the task-residual functional connectivity, which presumably reflects

333 information transfer, and displays certain levels of consistency with structural connectivity

334 (Bullmore and Sporns, 2009), and thus could potentially capture the direct

335 upstream/downstream areas for a seed area. Using these 29 areas as seed regions, we observed

336 fine-grained connectivity patterns for each seed region, showing hemispheric symmetry. These

337 areas were highly interconnected, and were also connected to the action-observation-related

338 areas (EBA, FBA, IPS, pSTS, SMA, PMd, PMv, M1, cerebellum), and DMN areas (dmPFC, vmPFC,

339 precuneus, TPJ, mSTS). Furthermore, most areas were heavily connected to bilateral caudate,

340 putamen and thalamus; and 14 out of 29 areas were connected to the hippocampus. See

341 supplementary Figure $\mathbf{S 6}$ for a summary of their connectivity patterns. 

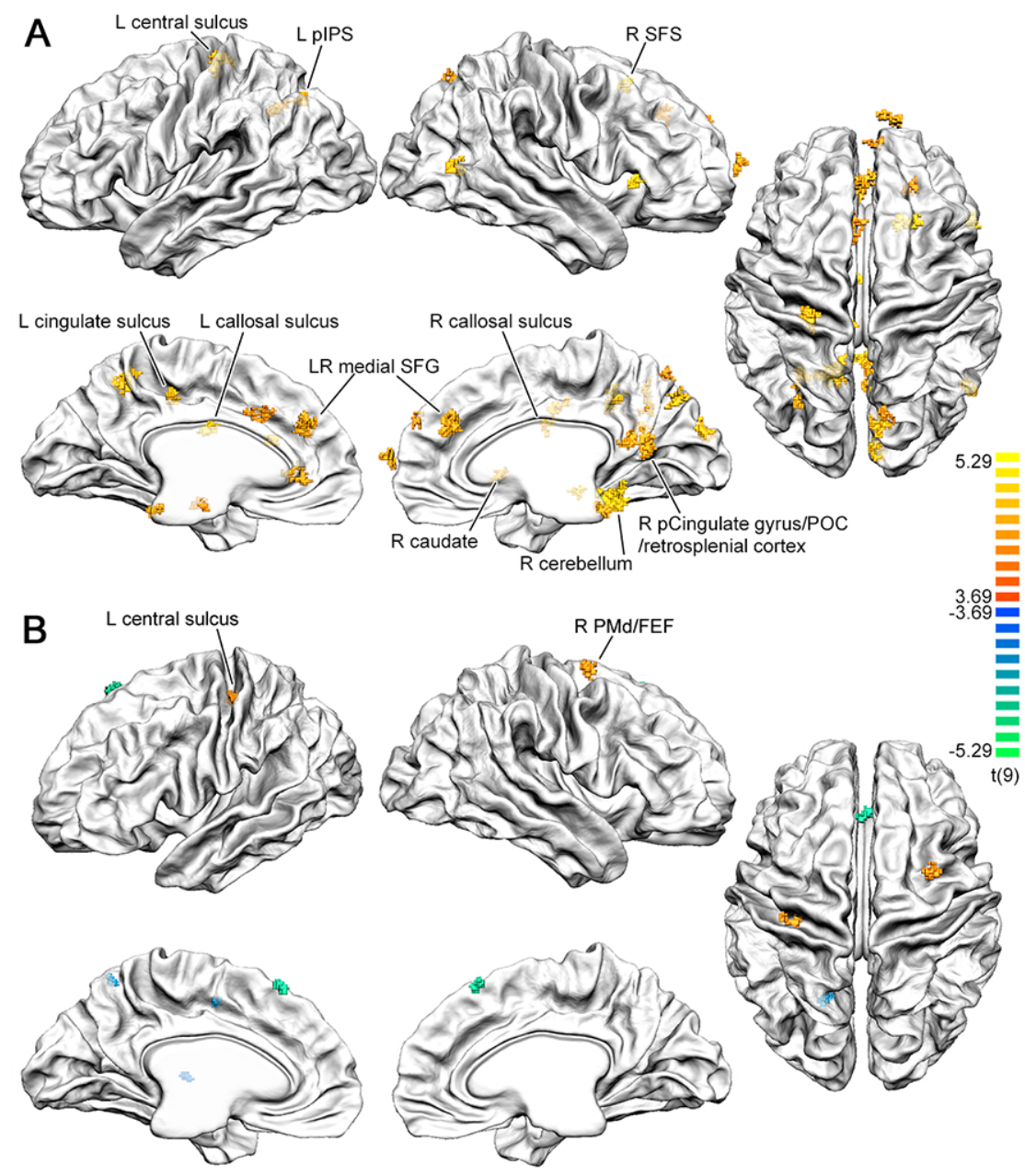

Figure 3. Searchlight RSA showed multiple areas correlated to the predefined category models.

A. Results for the 10 -action-category RDM, cluster size threshold=43 voxels. The labeled areas were ones overlapped with the perceived action and emotion FC networks. B. Results for the non-emotion/emotion RDM, cluster size threshold=35 voxels. The areas labeled were ones with positive correlations to the RDM. Color bar $p$ range in both A and B: 0.005 to 0.0005 . Cluster size thresholds: 43, 35 functional voxels. Initial $p<0.005$.

\section{The representations of low- or mid-level visual features and higher-}

\section{level attributes}

352 We used the predefined 10 action categories to search for brain areas with corresponding

353 "category boundaries". However, these category boundaries in the brain may not only be 354 generated by higher-level abstractions between categories, but could also be generated by lower-level visual feature differences between the stimuli. For our stimuli set, since the actors received a fixed set of instructions for performing the actions (Stienen and de Gelder, 2011), the postures may be somewhat stereotypical (see all stimuli in Figure S1), where low- or mid-level 
visual features alone could contribute to clear category boundaries, but co-vary with the abstract categories. Therefore, some of the brain areas found by the 10-action-category RDM may correspond to the low/mid-level visual features.

To examine this possibility in the 10-action-category areas found by searchlight, we performed RSA regression, using the RDMs of higher-level stimuli attributes and low-or mid-level visual features as predictors. The higher-level stimuli attributes included non-emotion/emotion, implied motion, valence, and actor identity. The low level features included the raw pixel values of each stimulus picture, and the body joint coordinates, extracted from each stimulus picture by the OpenPose library (Cao et al., 2019). The mid-level visual features were computed from the body joint coordinates and based on suggestions from the literature: The body-part orientations may signal the directions of social interactions; the hands/feet-to-head distances may be related to the peripersonal space (Bufacchi et al., 2016) and the relaxation of the arms/legs. Most of the low- mid-level RDMs indeed correlated to the 10-action-category RDM (rho ranging from 0.08 to 0.36 ), apart from the raw pixel value and head orientation RDMs. See Figure 4A, B. We acknowledge that these RDMs were only a very limited set from an infinitely vast model space. All RDMs were put in the same linear model, so that the unique contribution of each RDM could be examined.

We found that some of these stimulus features and attributes could partially explain the activity in some of the 10-action-category brain areas. For the low-level features, the raw pixel values representation could be found (with a positive beta estimate significantly bigger than 0 ) in $R$ cuneus and R CCZ, but showed a negative beta estimate in R SPOC; the raw joint coordinates representation could be found in R EBA, but showed a negative beta estimate in $\mathrm{R}$ callosal sulcus. For mid-level features, the head orientation representation could be found in R SPOC, while showing a negative beta estimate in $\mathrm{R} \mathrm{dmPFC}$; the shoulder orientation representation was found in L pIPS, and the shortest hand-head distance representation in $\mathrm{R}$ cerebellum. For higher-level attributes, only the actor identity representation showed a positive beta estimate in again the R SPOC, and a negative beta estimate in $\mathrm{R}$ cuneus. RDMs, which may either correspond to a true negative, or a false negative due to again the inter-individual anatomical variability. The implied motion rating RDMs showed positive clusters in L FEF (adjacent but not overlapping with the L PMd cluster for perceived emotion) and $R$ SPOC. And interestingly, the actor identity RDM showed a positive cluster in L MTS, close to EBA and FBA.

We found positive clusters for two mid-level visual features: head orientation and shortest

393 hand-to-head distance, but no positive clusters for any other low- or mid-level visual features, 394 despite their similarity to the two features with positive clusters (shoulder and waist orientation 395 RDMs were correlated to the head orientation RDMs: rho ranges from 0.317 to 0.412 ; the 396 shortest hand-to-head distance RDMs were correlated to the average hand-to-head distance 
bioRxiv preprint doi: https://doi.org/10.1101/2021.04.15.439961; this version posted April 15, 2021. The copyright holder for this preprint (which was not certified by peer review) is the author/funder, who has granted bioRxiv a license to display the preprint in perpetuity. It is made available under aCC-BY-NC-ND 4.0 International license.

397 RDMs, rho $=0.593,0.646$ for stimuli set $A$ and $B)$. This indicated that these two features may be 398 biologically meaningful ones for the brain.

A
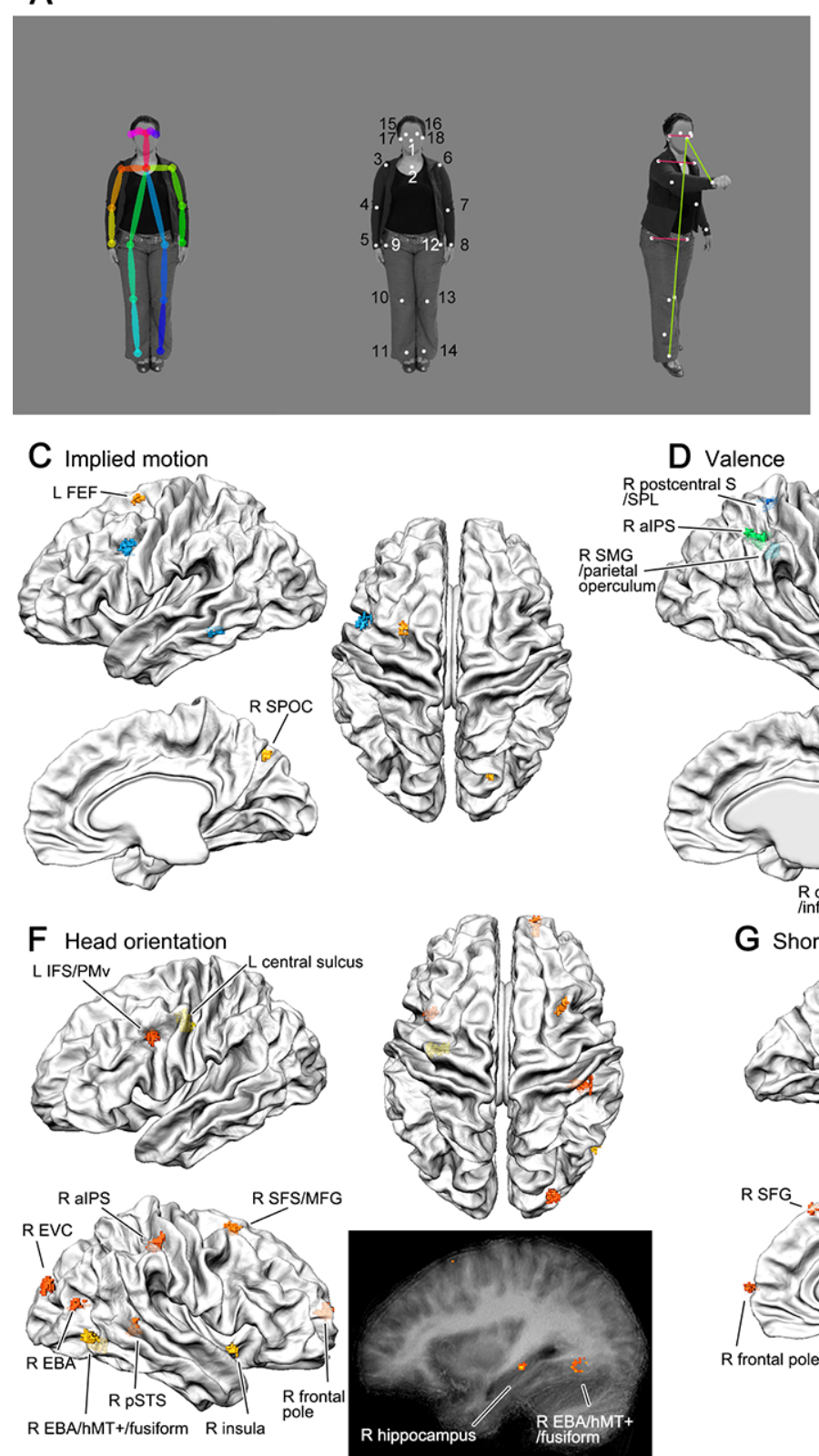

Shortest hand-to
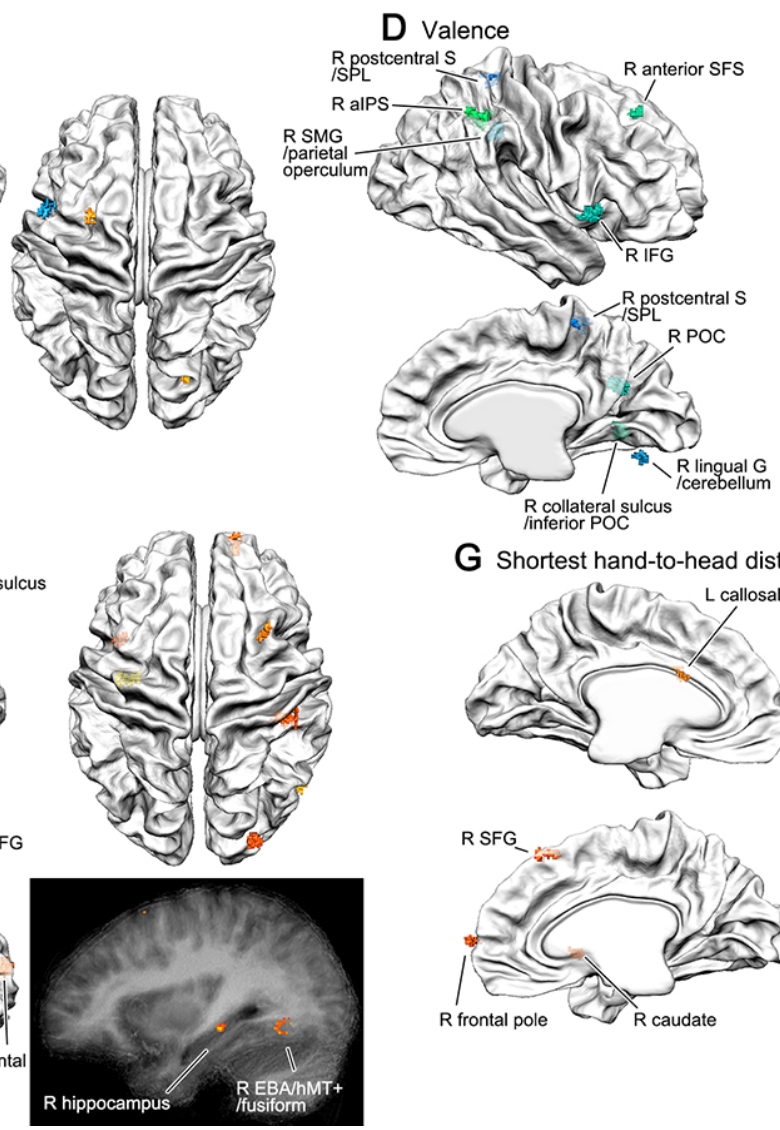

B

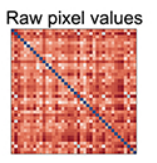

Head orientation
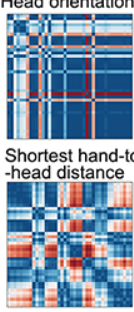

G Shortest hand-to-head distance
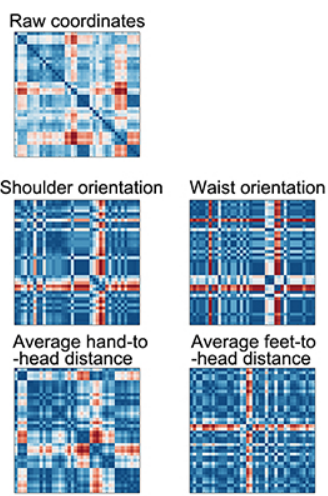

E Actor identity
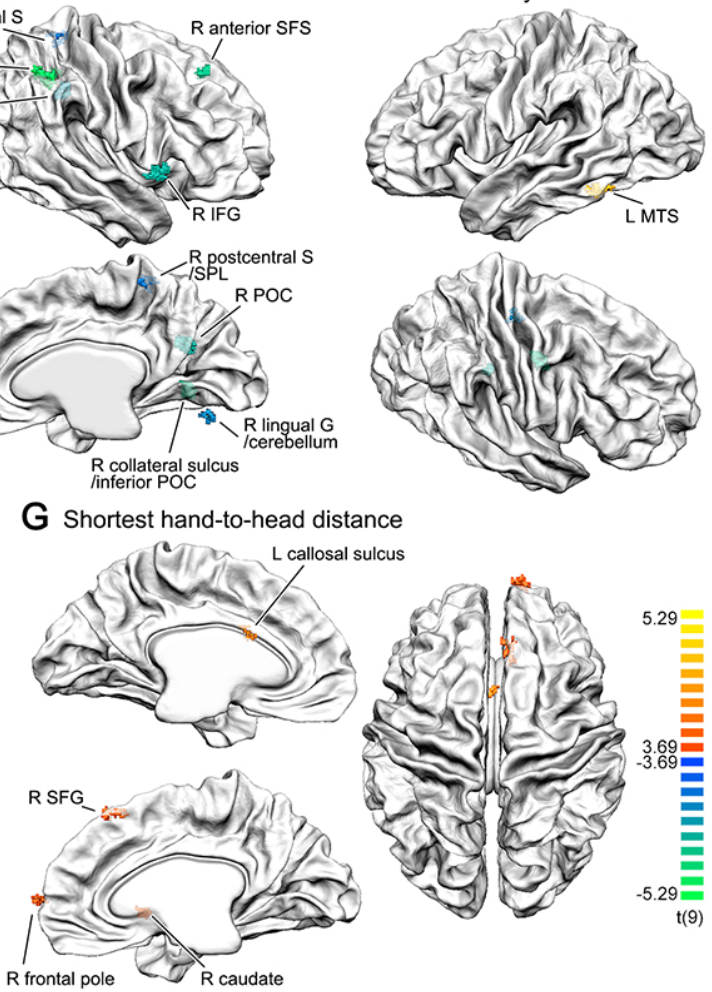

Figure 4. Low/mid-level features ( $\mathbf{A}$ and $\mathbf{B}$ ), and whole-brain searchlight results corresponding to these features ( $\mathbf{C}$ to $\mathbf{G}$ ).

A. Skeleton in the left panel: coordinates of the 18 joints extracted by the OpenPose library; white dots in the middle panel: joint coordinates after manual adjustment; right panel, magenta lines: distances used to compute head, shoulder, waist orientations, normalized by the corresponding distances in the neutral stand-still stimulus of the same actor (see middle panel); green lines: hand/foot-to-head distance. 
408 computed excluding joints on the ears and eyes, but was very similar to the ones computed

409 including these joints (RDM similarity rho $=0.976,0.98$ for stimuli set $A$ and $B$ ). Most of these

410 RDMs correlated to the 10-action-category RDM.

411 C, D, E. Searchlight results for higher-level attributes. The valence RDM did not show positively

412 correlated clusters. Cluster size thresholds: 38, 44, 31 functional voxels.

413 F, G. Searchlight results for mid-level features that showed clusters positively correlated to the

414 corresponding RDMs. Cluster size thresholds: 48, 37 functional voxels.

415 Color bar $p$ range in $\mathbf{C}$ to $\mathbf{G}: 0.005$ to 0.0005 . Initial $p<0.005$. Clusters showing positive

416 correlations to the corresponding RDMs were labeled. In $\mathbf{D}$, clusters showing negative

417 correlations (fine-grained processing with similar valence rating) were also labeled. 


\section{RSA searchlight analysis revealed representations for perceived action} 420 in left mSTS and for perceived emotion in left PMd

421 We next turned to the individual subjective reports, and searched for neural representations

422 similar to the individualized perceived action and emotion RDMs. We found one positively

423 correlated cluster for each of the subjective report RDMs, in left mSTS for perceived action, and

424 in left PMd for perceived emotion, indicating that these two areas may be involved in subjective

425 understanding and reporting for action and emotion, respectively. The L PMd cluster is

426 consistent with the analysis based on predefined non-emotion/emotion representation, where

427 the R PMd/FEF cluster was at a very similar location on the other hemisphere to the PMd cluster

428 found here.

429 All other clusters were negatively correlated to the subjective category RDMs, indicating fine-

430 grained processing for similarly perceived actions or emotions within those clusters. For

431 perceived action, the negative clusters include L RCZ and R supplementary motor area (SMA),

432 which were implicated in action observation in the literature (Caspers et al., 2010). For

433 perceived emotion, the negative clusters included R SMG/aIPS and R cerebellum for action

434 observation, $R$ insula for emotion processing, and mentalizing areas of $R$ precuneus, $R$ vmPFC, $R$

$435 \mathrm{dmPFC} /$ medial superior frontal gyrus (mSFG). See Figure $\mathbf{5}$ and TableS4 for the complete list of

436 clusters.

437 The L mSTS and L PMd clusters did not show reliable univariate activation for the 10 action

438 categories (one-sample t test of percent signal change against baseline, all $p>0.052$, except in $\mathrm{L}$

439 PMd for the Anger category, $p=0.031$ ), thus they could not be found by conventional univariate

440 analysis. Neither was the 10-category univariate ANOVA significant in these two clusters,

$441 p=0.309$ and $p=0.945$ respectively. 
442
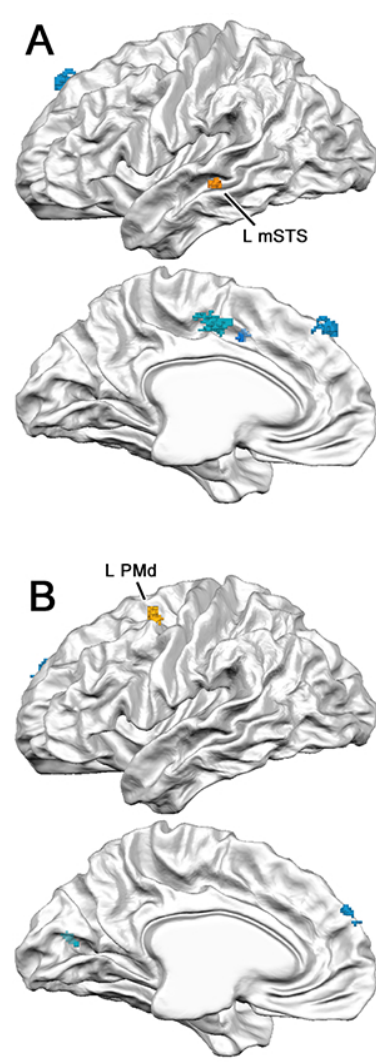
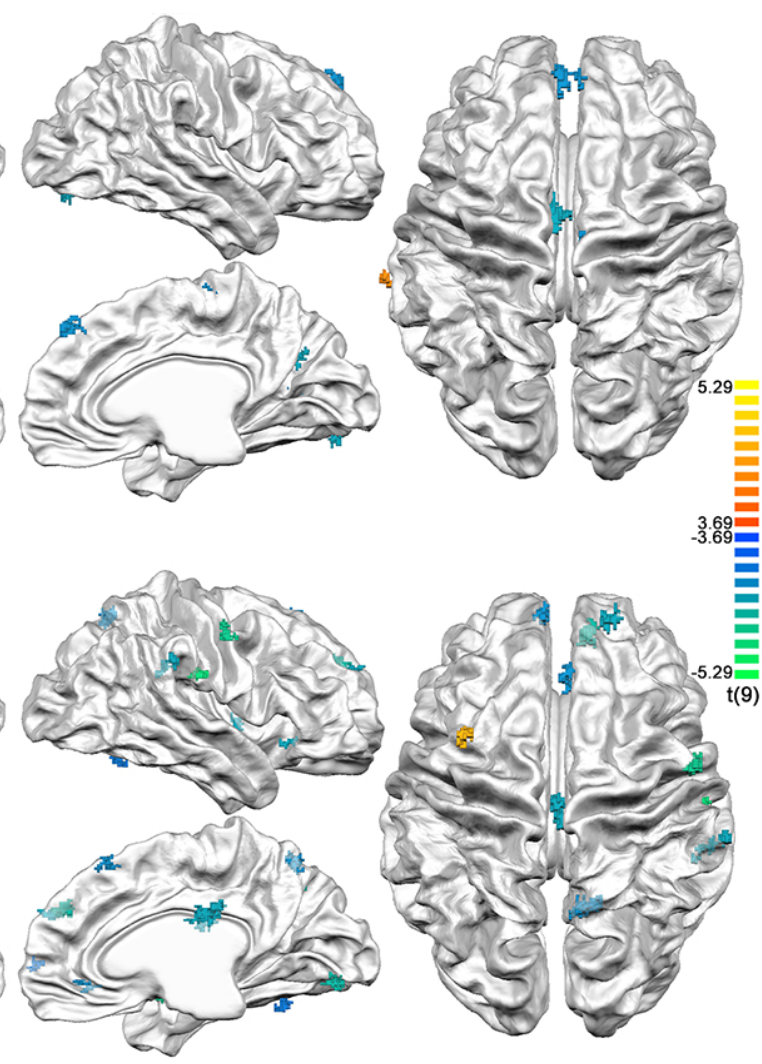

Figure 5. Searchlight RSA revealed corresponding clusters to the perceived action model in $\mathrm{L}$ mSTS (A), and to the perceived emotion model in L PMd (B). Color bar p range: 0.005 to 0.0005 . Cluster size thresholds in A and B: 39, 38 functional voxels. Initial $p<0.005$.

The putative direct upstream/downstream areas of L mSTS and L PMd identified by task residual functional connectivity and hierarchical clustering

The L mSTS and L PMd clusters were found by their representation similarity to individualized, subjectively reported actions and emotions, which already showed considerable individual variability. Thus, to further understand the involvement of these two areas in the current task, we could no longer use the standard predefined-model comparison methodology, which compares neural RDMs to a same model RDM, assumed by the researcher. Instead, we used a data-driven approach based on two assumptions: (1) We could potentially capture the information transfer stages of a certain area by functional connectivity; (2) Based on the idea that the object representations were successively re-represented and "untangled" in the ventral visual pathway (DiCarlo and Cox, 2007; DiCarlo et al., 2012), we assumed that this would be the case for bodily action and emotion representations throughout the brain, that the information (in our case the neural representations) should be gradually transformed across a chain or network of brain areas, from the representation of the visual input, to the representation of the subjective output, without sudden changes between two adjacent stages in the information- 
transfer chain. In this way, we linked the functional connectivity and representational analyses together (also see Ju and Bassett, 2020). To find the set of brain areas in the same informationtransformation chain, we performed task-residual functional connectivity to the L mSTS seed and the L PMd seed, respectively. Using hierarchical clustering in the two resulting networks, we could then find areas with most similar neural representations to the two seeds, which may include their direct upstream/downstream areas.

\section{9}

470

471

472

473

474

475

476

477

478

479

480

481

482

483

484

\section{Task-residual functional connectivity}

We regressed out the individual stimuli and head motion parameters from the functional data (deconvolution, data smoothed at $3 \mathrm{~mm}$ FWHM), obtained task-residual time courses, and performed functional connectivity (FC) analysis (Pearson's correlation), with L mSTS and L PMd as seed regions. See Figure 6A, B, left panels. This analysis revealed two partially overlapping FC networks for action/emotion understanding at the group level (Figure 6D), in action observation related areas including (bilateral if unspecified) IPS, M1, PMd, PMv, L IFG; and in DMN areas including TPJ, mSTS/MTG, precuneus, posterior cingulate cortex, dmPFC/vmPFC, mSFG, parietal occipital cortex (POC), retrosplenial cortex.

Interestingly, some overlapping areas in these $\mathrm{FC}$ networks are part of the semantic network, including $\mathrm{mSTS}$, IFG, TPJ/angular gyrus, dmPFC, vmPFC, posterior cingulate cortex, retrosplenial cortex (Binder et al., 2009); and the L mSTS FC network corresponded to the semantic network especially well. See Figure 6D and E. The seed cluster L mSTS itself is an important area in the semantic network, which could be activated by written word stimuli (Binder et al., 2009).

Although word-specific clusters around left mSTS were found in individual participants in the functional localizer data (words>other categories), neither the seed region nor the more extended FC cluster around the seed showed consistent group-level activation for words in the functional localizer (one-sample t test against 0, L mSTS seed ROI: mean beta=0.589; $t(9)=1.418$; $\mathrm{p}=0.190$; L mSTS FC cluster: mean beta $=0.369 ; \mathrm{t}(9)=1.226, \mathrm{p}=0.251$ ), which could again be due to the inter-individual anatomical/functional variability. More interestingly, although both FC networks overlapped at the L mSTS, the cluster for perceived emotion was much more posterior than the one for the perceived action, which corresponded to previous findings that the STS is an heterogeneous structure with several different functions (Hein and Knight, 2008).

In addition to the cortical clusters, the $\mathrm{FC}$ analysis revealed bilateral caudate and putamen clusters in both FC networks (Figure S7, S8), which may be involved in categorization processing (Seger, 2008; Seger and Miller, 2010). The left mSTS was further functionally connected to the R hippocampus; the left PMd was further functionally connected to the bilateral thalamus, pulvinar, cerebellum, the R septal nuclei, and the bilateral red nuclei (anatomical locations clearly observable in the $\mathrm{T} 2$ *-weighted functional images, Figure S8). The involvement of the hippocampus, the septal nuclei and the red nuclei in bodily action and emotion understanding were not routinely observed in previous bodily-action perception studies with univariate methods. 
501 The perceived action and emotion FC networks also overlapped with some of the clusters that negatively correlated to the perceived action/emotion RDMs in the searchlight analysis (Figure 3). They include R mSFG in the perceived action FC network; R posterior cingulate gyrus/callosal sulcus, R EVC, R lingual gyrus/cerebellum in the perceived emotion FC network. This indicates that subjective category-like processing and fine-grained within-category processing could be mediated within the same networks.

Furthermore, the perceived action FC network overlapped with the 10-action-category clusters in the L central sulcus, R POC/retrosplenial cortex and bilateral callosal sulci; while clusters in the perceived emotion FC network overlapped with the 10 -action-category clusters in L precuneus, $\mathrm{L}$ pIPS, L cingulate sulcus, R SFS, R posterior cingulate/POC/retrosplenial cortex, $\mathrm{R}$ caudate, $\mathrm{R}$ cerebellum and bilateral medial SFG (labels in Figure 3), again showing consistency to the

\section{Hierarchical clustering}

515 We then examined the possible direct upstream/downstream areas of the two seed regions, which should show the most similar neural representations to the seeds. We performed hierarchical clustering to all clusters within each FC network, on the group-averaged secondlevel neural RDMs across areas (Spearman correlation distance, hierarchical cluster linkage arbitrarily thresholded at 0.8 ). Since both seed regions were encompassed by more extensive clusters with the shortest hierarchical clustering distance to the seed (cluster in the perceived action FC network: spanning L MSTS, MTG and ITS; cluster in the perceived emotion network: an extensive one spanning LPMd, PMv and LR medial SMG), we plotted the extensive clusters (denoted as "seed clusters" below) instead of the seed regions themselves, in the dendrograms and second-level RDMs. See Figure 6A, B, Figure S9.

In the perceived action FC network, the FC cluster with the most similar representation (shortest distance in the dendrogram) to the seed cluster was one spanning L p/mIPS, TPJ, angular gyrus. The other areas under the same branch of the dendrogram were: the R mSTS contralateral to

528 the seed; action-perception related areas including bilateral PMv, MFG, L PMd, L IFG; DMN/semantic areas including $L$ precuneus, R TPJ, L posterior cingulate gyrus, LR dmPFC, LR and medial SFG.

537 These clusters may be directly involved in understanding the bodily actions and emotions. In 538 both FC networks, it is interesting to observe the strong involvement of both action-perception 
related areas and DMN/semantic areas, showing symmetry across hemispheres. Especially for the DMN/semantic areas, their direct involvement in bodily action/emotion understanding and interactions with the action-perception related areas were not reported in previous studies. This may be due to the observation that most of the areas in the two FC networks did not show an activation level different from baseline (one-sample t-test of beta values per action condition against baseline, FDR corrected across the 10 action categories per ROI). See Figure 6A, B, C. Thus, they were not likely to be localized when using only univariate methods.

The clustering in individual participants was relatively consistent, with moderately correlated second-level matrices across participants (averaged Spearman Rho, perceived action: 0.418, $\mathrm{SD}=0.0473$; perceived emotion: $0.374, \mathrm{SD}=0.0578$ ). See Figure $6 \mathrm{~F}$. Apart from the clustering

549 showing high similarity to the seed clusters in every participant (upper-left side of each RDM),

550 there appeared to be another prominent clustering of areas in each of the two FC networks of 551 individual participants (27-32 ${ }^{\text {nd }}$ areas in the perceived action FC RDM, 36-51 $1^{\text {st }}$ areas in the 552 perceived emotion FC RDM, both in the middle of the RDMs, see an enlarged version in Figure 553 S9), although less similar to the two seed clusters (red shade for FC clusters in Figure 6A, B, left panels). The clustering in the perceived action FC network consisted of areas around the bilateral central gyrus (lateral-central/central sulcus, post-central gyrus, bilateral SMA), most showing above-baseline activation for 0 to 3 of the 10 action categories, apart from the L lateral central sulcus showing activation for 8 categories. The clustering in the perceived emotion FC network consisted of areas in the bilateral ventral-lateral visual pathway (EVC, lingual gyrus, collateral sulcus, fusiform, EBA/hMT+, lingual gyrus, cuneus), where 8 areas showed abovebaseline activation for all 10 action categories, another area showing activation for 8 categories, the other 7 areas showing no activation for any of the categories. Despite the very strong differences in their univariate activation levels, their pattern similarity were clustered in the same branch of the dendrogram. Also, the second-level pattern-similarity clustering structure across areas does not seem to be linked to the low-level visual features from the stimuli, but seem to be a real organization feature, because the clustering structure could be seen in all 10 participants, despite the fact that each 5 participant saw a different stimuli set (set A and B). For these two groups of clustered areas, their further dissimilarity distance to the seed regions 569 seed regions, and our two assumptions of the information-transfer-chains seems to be able to 570 discover meaningful and replicable functional organizations in the brain. 
bioRxiv preprint doi: https://doi.org/10.1101/2021.04.15.439961; this version posted April 15, 2021. The copyright holder for this preprint (which was not certified by peer review) is the author/funder, who has granted bioRxiv a license to display the preprint in perpetuity. It is made available under ACC-BY-NC-ND 4.0 International license.
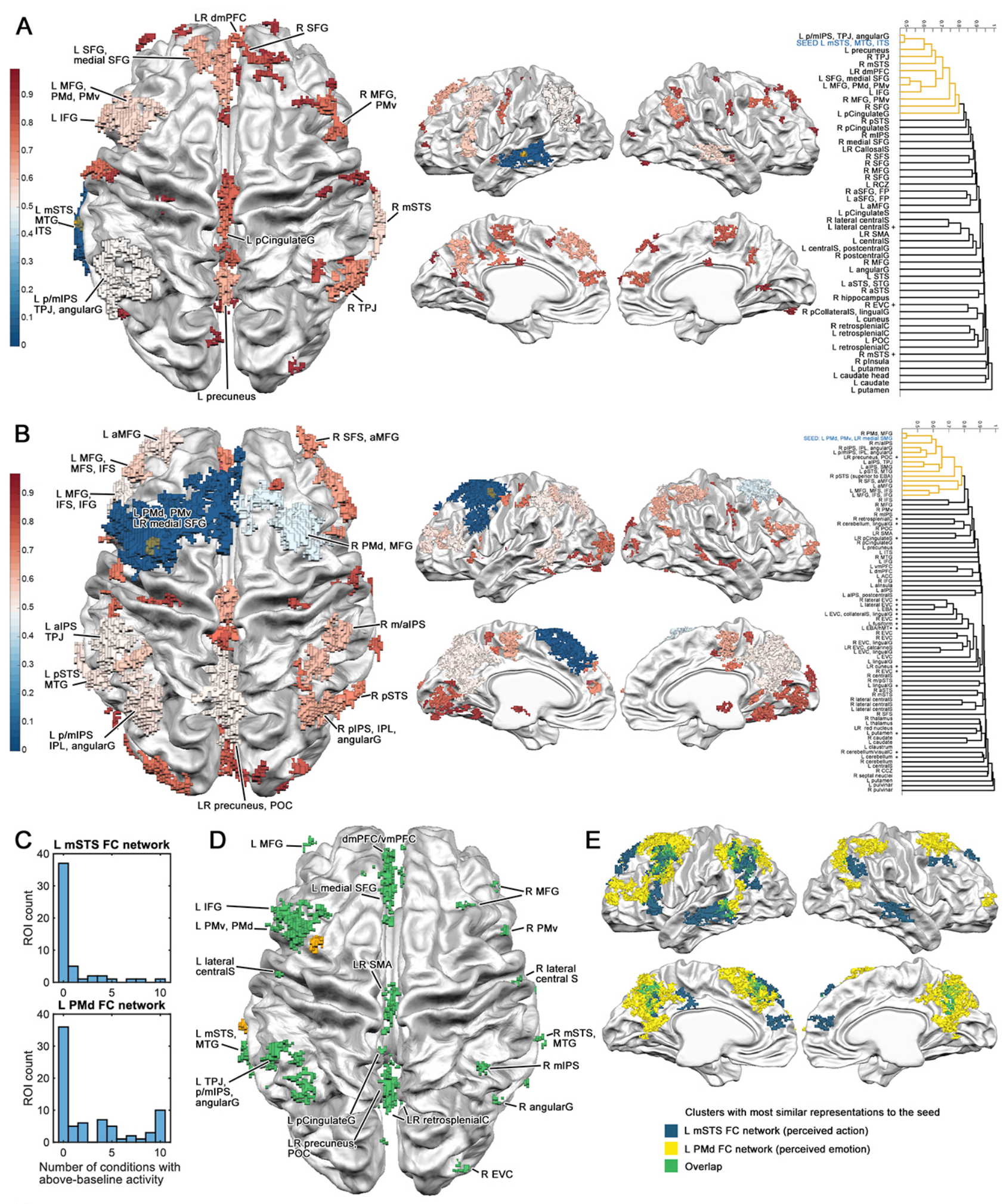

Clusters with most similar representations to the seed

$\square$ L mSTS FC network (perceived action)

L PMd FC network (perceived emotion)

Overlap

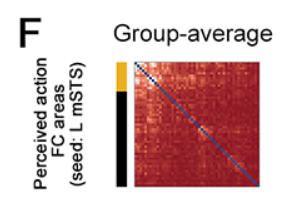

Individual
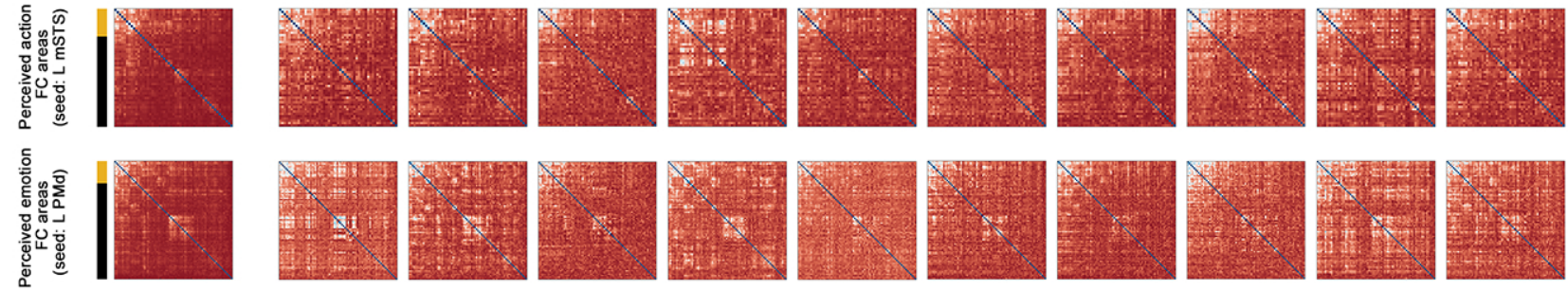
573 Figure 6. In the functional connectivity (FC) networks of the two seed regions overlapped with 574 the DMN/semantic networks.

575 A and B. Areas in the FC networks colorcoded by pattern similarity to the seeds, L mSTS (A), and

576 L PMd (B). Initial p<0.001, cluster size threshold in functional voxels: L mSTS network: 32; L PMd

577 network: 40 . The seed ROls were in yellow. Color bars: representational similarities to the FC

578 cluster containing the seed region. For each network, the hierarchical clustering dendrograms

579 computed on group-averaged second-level RDMs across areas (Spearman distance) were

580 plotted in the right-most panels. The areas with the most similar representations to the FC

581 cluster containing the seed region were colored in yellow in the dendrograms (linkage distance

582 arbitrarily thresholded at 0.8 , see a larger version in Figure S9), and labeled in the brain maps of

583 the left panels. The name of the areas containing the seed regions were marked in blue. The

584 areas with above-baseline univariate activation for more than 5 action categories were marked

585 with a "+" sign.

586 C. Histograms of number of conditions with above-baseline activity, for clusters in the two

587 networks. Most areas did not show univariate activation for any of the 10 action

588 categories/conditions.

589 D and E. The two FC networks overlapped with the DMN/semantic networks.

590 D. The overlap of two FC networks.

591 E. The overlap of the two FC networks, only showing clusters with most similar representations

592 to the seed (the clusters labeled in $\mathbf{A}$ and $\mathbf{B}$ ).

593 F. The group-averaged and individual second-level RDMs. The yellow and black bars for the

594 group-average RDM denote the same clustering of the dendrogram in A and B. See a larger

595 version in Figure $\mathbf{S 9 .}$

\section{Discussion}

598 In the current 7T fMRI study, we examined how participants perceived bodily actions, by

599 analyzing participants' subjective understanding of the actions and emotions displayed, besides

600 the conventional analysis by the predefined action and emotion categories. Dimension

601 reduction (PCA) revealed that subjectively perceived action and emotion representations were

602 high-dimensional and could not be reduced to the predefined category representations, despite

603 being correlated to them in smaller principle components. Some emotional categories were

604 reflected in the smaller principle components of the perceived emotion representation. Clusters

605 in L mSTS and L PMd had representations that were the most similar to the perceived action and

606 emotion representations. Areas located in the action-observation network and the semantic

607 network/DMN were functionally connected to these two clusters and also showed similar

608 multivariate patterns. This provided direct evidence for the involvement and interplay of both

609 networks in subjective understanding of bodily actions and emotions.

610 For the predefined categories, the non-emotion/emotion representation corresponded well

611 with the perceived emotion representation both behaviorally and neurally, showing a cluster in 
$612 \mathrm{R} \mathrm{PMd} / \mathrm{FEF}$, contralateral to the cluster for perceived emotion. For the predefined 10-action-

613 category representation though, the resulting clusters were more numerous, and likely

614 confounded by low/mid-level visual features that could co-vary with action/emotion categories.

615 However, all of the clusters were strongly connected to both the action-observation network

616 and the semantic network/DMN, consistent with the perceived action results. For mid-level

617 visual features, we further found that the head orientation and the shortest hand-to-head

618 distance were represented in the brain, indicating that these two features may be important for

619 the brain.

620 The involvement of the DMN/semantic network in both action and emotion 621 understanding

622 At first sight, it may seem that the involvement of the DMN/semantic network found in the

623 current study simply results from the use of word embeddings. We argue that this is not the

624 case, and that our study reveals the individual brain underpinnings of action and emotion

625 understanding as involving both the action observation network and the DMN/semantic

626

627 network. Although the word embeddings indeed represent the semantic distances between the words, these semantic distances reflect the similarities between different concepts. Our perceived action and emotion representations were different, despite being computed from word vectors from the same 300-dimensional space. Also, the perceived emotion cluster in L PMd is not a key node area in the semantic but rather in the action observation network; the two FC networks for perceived action and emotion did not completely overlap with each other either.

Actually, the involvement of IFG (Caspers et al., 2010; Dricu and Frühholz, 2016; de Gelder et al., 2004; Molenberghs et al., 2012) and areas in the DMN/semantic network (Chikazoe et al., 2014; Peelen et al., 2010; Skerry and Saxe, 2015) has been consistently found in action perception and emotional expression perception, although in separate studies and in different contexts. For the DMN and especially for the VMPFC area, their involvement in emotion perception was for abstract representation of emotional stimuli found with multivariate RSA (Chikazoe et al., 2014; Peelen et al., 2010; Skerry and Saxe, 2015), rather than with simple univariate contrasts. The simultaneous involvement of the IFG and the DMN in action understanding was found in one univariate study contrasting participant's attention on either the intention or means of the performed actions (de Lange et al., 2008). In that study, the IFG activity was higher for actions with unusual intentions compared to usual ones; the DMN was instead showing higher activity when participants were paying attention to the intentions rather than the means of the action.

Two recent studies provided more converging evidence to us. One RSA fMRI study about observed actions (Tucciarelli et al., 2019) used a large set of action categories, constructed a semantic similarity model of the meanings of the actions as well as other similarity models obtained from individual participants' behavioral sortings of similarities, and searched for corresponding RDMs in the brain. They found bilateral clusters in IFG/PMv, pIPS, and lateral occipitotemporal cortices (LOTC, close to EBA) corresponding to the semantic similarity model, although only the left LOTC cluster remained after RSA regression controlling the effects of 
other models. That study controlled the inter-individual consistency of the stimuli perception during the stimuli selection process, which was not done in the current study, and may explain the discrepancy. Another study (Masson and Isik, 2021) examined the fMRI responses during naturalistic movie watching using encoding models, and found that a social-affective model (including features of an agent speaking, social interactions, theory of mind, perceived valence and arousal) significantly explained the fMRI response in the left STS across two different movies. This cluster fully overlapped with our L mSTS cluster.

In fact, the IFG and the areas in the DMN were all parts of the semantic network, thus their involvements could also be studied in the future in the context of the semantic network, apart from the context of "mentalizing" usually associated with the DMN. The importance of the semantic network in emotion understanding is further supported by behavioral studies of semantic dementia patients. In one such studies, three semantic dementia patients with left temporal pole atrophy and impaired semantic knowledge were asked to sort faces into piles by emotion. These patients were not able to distinguish between emotional faces with negative valence, despite that their perception of positive/negative affect, the visual features for each facial emotion and identity were intact (Lindquist et al., 2014).

Although we did not examine the temporal pole due to coverage limitation, our multivariate RSA and functional connectivity results support the importance of the DMN/semantic network in action and emotion understanding. These results are also consistent with the literature, that the DMN did not show above-baseline activation, when passively observing actions (videos, non-social, non-emotional) but without deliberating the goals/intentions (Van Overwalle and Baetens, 2009). We further revealed that both the DMN/semantic network and the action observation network were involved in the process of action and emotion understanding, that they were consistently found in all 10 participants within the same functional connectivity networks and showed similar multivariate patterns despite very different levels of univariate activation. The multivariate methods worked in our study and in previous RSA studies, because they took the multiple dimensions in the high-dimensional data into account, while the univariate method considers only one or a few specific dimensions which associated with specific contrasts (Haxby et al., 2011). Our study stresses the importance to further examine the function of the DMN/semantic network in action and emotion understanding with multivariate methods in future studies.

\section{Understanding versus categorization}

Previous experiments have mostly used explicit emotion and action categorization tasks with predefined categories, and implicitly used explicit categorization as a proxy to study subjective understanding. However, categorization and understanding may involve different neural substrates, as categorization involves some level of abstraction. Our study could not differentiate between these two processes, because first, the RSA method could not disentangle categorical boundaries driven by lower-level visual features, or concrete perceptual categories (Hoemann et al., 2020; Mansouri et al., 2020) that were bound to individual exemplars, or abstract categories that generalize across exemplars. Second, what we obtained in the 
subjective reports were descriptions or labels of action and emotion for each individual stimulus, rather than more abstract categorization to the group of stimuli. Thus, the resulting $L$ mSTS and L PMd clusters for perceived action and emotion may not represent the most abstract level, and the more abstract categorization may happen in other areas functionally connected to these two areas, and could perhaps utilize these category boundaries in computation. The different levels of abstractions may also have driven the discrepancy between the analyses with subjective reports and predefined categories, although both analyses pointed to the strong involvement of the caudate, which is in the executive loop of categorization learning tasks (Seger, 2008; Seger and Miller, 2010). The areas found in our study could serve as target areas in future studies, to examine the level of abstraction.

\section{2}

\section{Brain areas for emotion understanding}

Our analysis with the predefined non-emotion/emotion category model and with the perceived emotion model pointed to the PMd areas in the right and left hemispheres. This area may contain infromation exhibiting categorical boundaries between non-emotional/emotional stimuli, but again the level of abstraction could not be disentangled. Future studies could use emotional facial or voice stimuli, accompanied by subjective reports, to examine whether this area is specific to bodies and actions, or is more general for emotions in different modalities (Vaessen et al., 2019a).

We did not find evidence for coding of valence in frontal (higher-order) areas, either with monotonic univariate activity modulation or with multivariate representations. However, valence coding may be bivalent, such that the vmPFC/mOFC activity monotonically increased for both positive and negative valence, as found in an RSA study (Chikazoe et al., 2014). This might be the case, as we found evidence that the VMPFC was showing a fine-grained pattern between similarly perceived emotions (Figure 5B). With only one category of positive emotion, we were not able to examine the possible bivalent activity. modulation of valence rating, we found adjacent/overlapping clusters in the $L$ central sulcus (Fig. 2D, Fig. 3). These clusters are also adjacent/overlapping to the two FC networks for perceived action and emotion (Fig. 6AB), but with different neural representations to the two seed regions for perceived action and emotion. This indicated that the primary sensorimotor areas were involved in action and emotion processing, although the representation there may be of lower-level features and may not be emotion-specific.

\section{Importance of using individualized subjective reports to study higher- 725 level cognition}

726 With subjective reports, we found that the perceived action and emotion representations were

727 more high-dimensional and multi-faceted than the predefined category representations,

728 consistent with the recent series of studies using subjective reports (Cowen and Keltner, 2017,

729 2020, 2021; Cowen et al., 2019). We also found neural representations corresponding to these

730 subjective report representations in higher-level areas outside the ventral and dorsal pathways. 
731 Apart from a few RSA studies which linked individualized behavioral data to the brain data

732 (Chikazoe et al., 2014; Stolier and Freeman, 2016; Tucciarelli et al., 2019), multiple previous RSA

733 studies either utilized only the predefined categories (Peelen et al., 2010), or the behavioral

734 ratings from an independent group (Bracci and Op de Beeck, 2016; Mur et al., 2013; Peelen et

735 al., 2014; Skerry and Saxe, 2015; Vaessen et al., 2019b), or the averaged behavioral responses of

736 the same participants scanned (Connolly et al., 2012; King et al., 2019). For high-level cognition

737 that has a more remote relation with the sensory stimuli and has more variability between

738 individual participants, the use of individualized behavioral data may be beneficial, as was

739 evidenced for studying object recognition (Charest et al., 2014), which was traditionally thought

740 to have considerable behavioral judgment similarities shared between participants. The multiple

741 pre-trained word embeddings are also relatively easy to use and enable objective analyses of

742 subjective reports.

\section{Advantages and limitations of the current 7T experiment}

744 The use of high-resolution 7T fMRI in the current study has advantages and limitations. With

745 higher gray-white-matter contrast and higher temporal signal-to-noise ratio, the resulting data

746 were very robust (see the functional localizer data in Figure S2 and S3), the activation clusters in

747 individual participants were highly localized in the gray matter, and we observed small

748 subcortical clusters in multiple analyses at the group level, including periaqueductal gray, medial

749 geniculate nucleus, substantia nigra, red nucleus, and the septal nuclei. However, the current 7T

750 data also induced the possibility of false negatives, where the inter-individual

751 anatomical/functional variability was exacerbated and would no longer be compensated by

752 extensive smoothing, as observed again in the functional localizer data (Figure S3). Better

753 whole-brain group-level analysis schemes apart from the ROI analysis are needed to benefit

754 from both the high functional resolution and the large brain coverage, which potentially include

755 more fine-grained functional parcellations (Glasser et al., 2016; Yeo et al., 2011) and

756 hyperalignment (Haxby et al., 2011).

\section{Acknowledgments}

759 This study was supported by the European Research Council, under the European Union's

760 Seventh Frame-Work Programme (FP7/2007-2013)/ERC (grant numbers 295673 to BdG and

761269853 to RG), by the ERC-Synergy program grant RELEVANCE (Grant agreement 856495 to

762 BdG), and by the FPN-MBIC funding of Maastricht University to MZ and BdG. MZ was supported

763 by Fondation Bettencourt Schueller. We thank Federico de Martino for setting up the 7T

764 scanning sequences and Giancarlo Valente for providing a part of the searchlight code. We

765 thank Maarten Vaessen for commenting on a previous version of the manuscript. 


\section{Author Contributions}

767 Conceptualization: M.Z. and B.dG.; Methodology, Software, Validation, Formal Analysis, 768 Investigation, Data Curation, Writing - Original Draft, Visualization: M.Z.; Resources: R.G.;

769 Writing - Review \& Editing: M.Z., R.G. and B.dG.; Project Administration, Funding Acquisition:

770 M.Z. and B.dG.; Supervision: B.dG.

\section{Declaration of Interests}

772 The authors declare no competing interests.

\section{Data and code availability}

774 The data and codes of this study are available at

775 https://osf.io/cuh9v/?view only=efb12b7585ee4b6bbcfd7ca42c63b60d, including: stimuli

776 images with body joint estimations, subjective reports and corresponding word embeddings,

777 whole-brain statistical result maps, ROI raw data for the RSA analysis, structural images and

778 white-gray-matter-boundary segmentations.

Materials and Methods

780 Participants

781 The data of 10 healthy right-handed participants recruited from the campus of Maastricht

782 University were included in the analyses (mean age $=23.4, \mathrm{SD}=1.955,5$ females.) Two more

783 participants took part in the study, but due to excessive head motion observed during the scan,

784 their scanning sessions were either aborted, or data excluded from the analyses. All participants

785 had normal or corrected-to-normal sight and had no history of psychiatric disorders. Participants

786 provided written consent before the study and received monetary reward afterwards. The

787 experiment was approved by the ethical committee of Maastricht University, and was carried

788 out following the declaration of Helsinki. The experiment was conducted in English.

\section{Data acquisition}

790 The MR data were acquired in a 7T Magnetom full-body scanner (Siemens, Erlangen, Germany)

791 in Scannexus, Maastricht University, with a Nova 1-transmitter/32-receiver head coil (Nova

792 Medical, Wilmington, USA). Dielectric pads were used for all participants except S10 (when the

793 head size didn't allow it), roughly covering bilateral occipito-temporal lobes. The stimuli were

794 back-projected onto a screen behind the participants' head (Projector: Panasonic PT-EZ57OEL,

795 projected screen size $30 \times 18 \mathrm{~cm}$, resolution $1920 \times 1200$ pixels, refresh rate $=60 \mathrm{~Hz}$, viewing

796 distance $\sim 99 \mathrm{~cm}$, screen visual angle $17.23 \times 10.38$ degrees) and the participants viewed the

797 screen through a mirror fixed on the head coil. Participants came for two scanning sessions, a 2-

798 hour session for the main experiment, and a 1-hour session for functional localizers and

799 anatomical scans. 
800 Whole-brain anatomical data were collected for each participant with a resolution of $0.6 \mathrm{~mm}$ 801 isotropic (MPRAGE sequences, FOV $=229 \times 229 \mathrm{~mm}^{2}$, matrix size $=384 \times 384$, flip angle $=5$. T1-

802 weighted: TR=3100 ms, TE=2.52 ms; proton-density-weighted: TR=1440 ms, TE=2.52 ms). For 803 the functional runs, a 2D gradient-echo multi-band EPI sequence was used, with a resolution of $8041.2 \mathrm{~mm}$ isotropic (multi-band acceleration factor=2 (Moeller et al., 2010), iPAT=3, FOV=172.8 $\mathrm{x}$ $805172.8 \mathrm{~mm}^{2}$, matrix size $=144 \times 144$, flip angle $=75$, number of slices=70, slice thickness $=1.2 \mathrm{~mm}$, 806 no gap, ascending interleaved 2, TR=2000 ms, TE=21 ms, encoding direction Anterior to 807 posterior, reference scan mode: GRE, MB LeakBlock kernel: off, fat suppression enabled). In 808 each scanning session, a head scout was acquired for localization, then the B0 field map was 809 acquired and loaded in the console. The interactive shimming was performed before acquiring 810 the B1 field map. The system voltage was then computed according to the B1 map values (set to 811 a maximum of $190 \mathrm{~V}$ across all sessions), to have a 90 degree flipping angle at the white matter 812 beside the lateral ventricles, and the specific absorption rate (SAR) level for the longest

813 functional run (432 volumes) was controlled at below $75 \%$. The slices were tilted in an angle that 814 covered most of the occipital lobe, parietal lobe and frontal lobe, while leaving out the anterior 815 temporal lobe, part of the motor cortex, and the orbitofrontal cortex. This was to ensure that 816 most of the important areas involved in body perception were covered, including EBA, fusiform 817 gyrus, IPS, IPL, PMd, PMv. The amygdala was not consistently covered given the relatively 818 limited coverage (covered in 6 out of 10 participants). Immediately before each functional run, a 8195 -volume run of the same setup but with posterior to anterior encoding direction was acquired 820 (Invert RO/PE polarity: on), for post-hoc top-up EPI distortion correction (See the fMRI data 821 preprocessing sub-section). We informed the participants the purposes of these distortion 822 correction runs, and instructed them not to move between the distortion correction run and the 823 actual functional run.

\section{Stimuli}

825 The stimuli were gray-scale whole body images developed and validated in our lab (Stienen and 826 de Gelder, 2011). They consisted of 8 actors, each posing 10 actions with or without emotional 827 content. The first 5 categories were neutral actions: combing hair $(\mathrm{CH})$, drinking water (DW), 828 opening door (OD), talking on the phone (PH), putting on trousers (TR); the $6^{\text {th }}$ was neutral 829 standing still (NE), and the last 4 were emotional expressions: fear (FE), anger (AN), happy (HA), $830 \mathrm{sad}(\mathrm{SA})$. The 80 postures were split into 2 balanced sets by randomly selecting images from 4 831 actors for each category, resulting in 2 sets of 40 stimuli (4 images per category, 5 images per 832 actor), which ensures that in each stimulus category the participants perceive as much 833 variability in the posture and the identity as possible. Each participant saw one of the sets.

834 The body stimuli were embedded a gray background ( $R G B$ value $=128,128,128$ ), with all 835 internal facial information removed. They were sized to $400 \times 600$ pixels, and presented 836 centrally on the screen ( $R G B$ value $=128,128,128)$. The whole-body shapes in the images 837 overall spanned $309 \times 492$ pixels on the screen (visual angles=2.60 $\times 4.26$ degrees). 


\section{$838 \quad$ fMRI experiment design}

839 The study used a slow event-related design. Stimuli were presented with Matlab (Version

840 R2012a, the MathWorks, Natick, USA) and Psychtoolbox 3.0.11 (Pelli, 1997). A white fixation

841 cross was present in the center of the screen throughout the experiment. The participants were

842 asked to always fixate on the fixation cross and take in the body posture as a whole. The

843 experiment consisted of 6 runs ( $14 \mathrm{~min} 18 \mathrm{~s}$ each, 429 volumes). Each run started with a fixation

844 period of 8 seconds, and then the whole set of 40 stimuli was presented to the participant twice

845 within each run. Each stimulus was presented for $500 \mathrm{~ms}$, followed by an inter-stimulus interval

846 of either $7.5,9.5$ or $11.5 \mathrm{~s}$. The stimuli and the ISI were presented in a pseudorandomized order.

847 In addition, 4 catch trials were included in each run. Within each catch trial, a body posture was

848 randomly drawn from the stimulus set, while the fixation cross changed to either red or blue

849 (RGB color red=195, 32, 30; blue=10, 109, 195) during the stimulus presentation period.

850 Participants were asked to indicate the color by pressing the corresponding button of the button

851 box as soon as they saw the fixation change color. Two seconds were added to the inter-

852 stimulus interval after each catch trial. Excluding the catch trials, each single stimulus image was

853 presented to the participant for 12 times throughout the main experiment. 9 participants

854 completed all 6 functional runs; 1 participant completed 5 runs.

\section{Functional localizers}

856 A separate scanning session was devoted to acquiring functional localizer data and the structural 857 images. Stimuli were presented under passive viewing condition using Presentation software 858 (Version 16.0, Neurobehavioral Systems, Inc., Berkeley, USA). In the static localizer run (14 min $85924 \mathrm{~s}, 432$ volumes), after a $12 \mathrm{~s}$ fixation period, gray-scale stimuli of faces, houses, bodies, tools 860 and words were presented in blocks of $12 \mathrm{~s}$ (12 stimuli per block, 800 ms stimuli presentation, 861200 ms inter-stimulus interval), followed by resting periods of $12 \mathrm{~s}$ with the fixation cross on a 862 blank screen (RGB value=157,157,157). Each category block was presented 7 times, with a 863 pseudorandomized presentation order for both the stimuli and the blocks. Facial stimuli were 864 front-view neutral faces from the Karolinska Directed Emotional Faces (Lundqvist et al., 1998) 865 (24 identities, 12 males). The part below the neck (clothes, hair etc.) was removed from the face 866 images. Body stimuli were neutral still front-view bodies (de Gelder and Van den Stock, 2011) 867 (20 identities, 10 males) from a different set than the one used in the main experiment, with the 868 facial information removed. House and tool images were obtained from the internet. The house 869 images consisted of 19 facades of houses with 2-to-3-storey height; the tool images consisted of 87018 hand-held tools; words images consisted of high-frequency English words of 4-6 letters in 871 Arial font. All the images were imbedded within a gray background (RGB value $=157,157,157$ ), 872 spanning a visual angle of 1.99 degrees (230 pixels).

873 The dynamic localizer run (5 min $36 \mathrm{~s}, 168$ volumes) consisted of 1-s video clips of either facial or 874 bodily expressions, including neutral (coughing or clearing throat), angry, fear, happy (Kret et al., 875 2011). The actors performed the actions against a green background, either wearing black 876 clothes for bodily expressions, or green clothes for facial expressions. Two exemplars were 877 selected for each expression category (in total 8 for faces and 8 for bodies). The actors in the 
878 selected exemplars were all males. For the facial stimuli, two of the neutral expressions were performed by the same identity. The facial and bodily expression clips were presented separately in blocks of $8 \mathrm{~s}$, with the stimuli order pseudorandomized. The face and body blocks were presented 10 times each, separated by an inter-block interval of $8 \mathrm{~s}$, where a black screen and a white fixation cross was presented.

\section{Behavioral ratings}

Right after the scanning session, participants completed a behavioral task outside the scanner. Each of the 40 stimuli participant saw in the scanner was presented once using Psychopy (v1.83.04)(Peirce, 2007) on an LCD monitor (Acer VG248, resolution $=1920 \times 1080$, refresh rate $=$ $60 \mathrm{~Hz}$, whole-body size in the stimuli spanning the visual angle of $13.42 \times 8.43$ degrees). For each stimulus image, 6 questions were answered either with a 7-point scale (for implied motion and valence respectively), or with an open answer with free typing (for the action the actor performed, and the emotion). See Table $\mathbf{S 1}$ for details of the questions. We also recorded whether the participants changed their perception during the scan (questions 2 and 3). Four participants changed their perceptions (S4 and S9 for 2 stimuli, S6 for 7 stimuli, S10 for 12 stimuli). We used only the initial perception in the scanner (answer for question 1) for subsequent analyses. The same stimulus image stayed on the screen for all 6 questions. Participants answered the questions at their own paces (mean time spent $=21.47 \mathrm{~min}, \mathrm{SD}=6.98$ min, range: 12.61 to $32.89 \mathrm{~min}$ ).

\section{Data analysis}

\section{Representational similarity analysis (RSA) for behavioral data}

We mapped the subjective reports with Deconf word embeddings (Pilehvar and Collier, 2016), which linked the Word2vec embeddings and the WordNet database. Word2vec embeddings were trained on a very large corpus of text, able to capture various linguistic relationships between words (Mikolov et al., 2013). After training, words with similar semantic meanings were found to be situated closer to each other in the embedding vector space (cosine distance); although for word2vec, different meanings of the same word were not disambiguated. On the other hand, WordNet (Miller, 1995) is a curated lexicon database, where different meanings of each single word were separated, and synonyms were grouped together; but it does not provide a quantitative mapping for word similarities. Deconf embeddings mapped the individual word meanings (senses) in WordNet into the 300-dimensional word2vec vector space, offering us both a common high-dimensional semantic space trained by a large corpus, and the precise separation of different meanings.

For behavioral free reports, we omitted the pronouns (e.g. he is, she is, his, her), as most of the times participants did not consistently type in the pronouns. The rest of the words/phrases (nouns, verbs, adjectives, adverbs) that had a corresponding entry in WordNet 3.1 were lemmatized (e.g. stretching $\rightarrow$ stretch). For words/phrases not found in WordNet 3.1, "how" was omitted; "something" was substituted by "thing"; "himself/herself/oneself" were substituted by "self". The adverb "just" associated with verb phrases was omitted (e.g. just watching $\rightarrow$ watch). 
917 When describing the perceived action, "not sure" was substituted by "unsure"; when denoting 918 that the participant had no idea about what the person was doing, "no idea" and "not sure" 919 were substituted by "not applicable". For words with multiple meanings ("senses" the term used 920 in WordNet), the corresponding sense was selected. When a noun denoting emotion has both 921 senses of <noun.feeling > and <noun.state>, the sense of <noun.feeling > was always selected.

922 There were very few cases that participants typed in "not". For one case the participant 923 denoting the emotion of the person in the stimuli "was not sure about something", it was 924 substituted with the word "unsure". All the other cases were in responses for perceived acitons, 925 about participant's own uncertain understanding for the stimuli. For these and similar cases we 926 substituted the entry with "not applicable". For all response entries and word lists, see 927 https://osf.io/cuh9v/?view only=efb12b7585ee4b6bbcfd7ca42c63b60d.

928 For each free report entry after lemmatization, the 300-dimension word vector with the 929 corresponding sense number and sense key were selected from the Deconf pre-trained 930 embeddings. When an entry has multiple words, the vectors were averaged for this entry. The 931 RDM for each participant were then computed in cosine distance, as this metric was routinely 932 used for computing word-embedding distances in the literature.

933 The RDMs for behavioral ratings (implied motion, valence) were computed directly from the 934 ratings, in Euclidean distance.

935 For predefined RDMs, each category was binary-coded in vectors, with numbers of elements 936 corresponding to the total number of categories. E.g. for predefined actions, drinking water $\left(2^{\text {nd }}\right.$ 937 category in 10) was coded as 0100000000 ; for predefined non-emotion/emotion 938 categories, emotional ones were coded as 01 . This coding assumes that each category was 939 orthogonal to the others. The resulting RDMs were computed in Euclidean distance.

940 For RDM comparisons throughout the study, the Spearman's correlation was computed 941 between $40 \times 40$ RDMs, with the 780 values below the diagonal of the RDMs as inputs. The 942 resulting rho values were Fisher's $Z$ transformed, submitted to a one-sample $t$ test against 0 943 (two-tailed) at the group level. The group-averaged $Z$ values were back transformed to rho 944 values (or 1 - rho distance) and reported. To assess individual variability, we computed the 945 coefficient of variation (CV, sample standard deviation/sample mean, in \%).

946 Since all 400 response entries for the perceived action and emotion representations were in the 947 common 300-dimensional word-vector space, we performed PCA on the 400 entries for each 948 representation (without centering, since the word vectors were all normalized). The PCA scores 949 for each principal component (PC) was computed into RDMs (Euclidean distance), and 950 correlated with $400 \times 400$ model RDMs. The model RDMs were created in the same way as the $95140 \times 40$ RDMs, with the only difference that the 400 entries were sorted by action categories 952 across all participants. Model RDMs included predefined action, predefined emotion, implied 953 motion, valence, individual subjects (entries of the same participant were coded as the same 
954 vector). The PCA scores of all response entries were plotted in the two PCs that showed the

955 highest positive correlation to the model RDMs (Fig. 1I, J).

957 fMRI data Preprocessing

958 The data were processed with BrainVoyager (version 20.0 and 20.2, Brain Innovation,

959 Maastricht, the Netherlands), MATLAB (version R2016a), and NeuroElf 1.0 toolbox implemented

960 in MATLAB (http://neuroelf.net/). Before any preprocessing, the functional data underwent in-

961 plane EPI distortion with the COPE plugin (v1.0) in BrainVoyager

962 (https://support.brainvoyager.com/brainvoyager/available-tools/86-available-plugins/62-epi-

963 distortion-correction-cope-plugin). The voxel-wise displacement was estimated between the

964 first volume of each functional run and the first volume of the preceding 5 -volume correction

965 run (in reversed phase encoding direction). The in-plane voxel-wise displacement map was then

966 applied to all the volumes of the functional run. The first volume of each run was saved as a

967 separate file, and applied with distortion correction. This volume then served as the basis for

968 subsequent motion correction and across-run alignments. After distortion correction, the

969 functional runs underwent slice scan time correction with the slice timetable information from

970 the scanner (interpolation: sinc), within-run 3D rigid motion correction with each run's first

971 volume as references (interpolation: trilinear for motion estimation, sinc for applying

972 transformations), and temporal high-pass filtering (GLM with Fourier basis set of 2 cycles,

973 including linear trend). The order of distortion correction and motion correction was assessed

974 on S06's sixth (last) main-task run, which has the biggest within-run rotation in all acquired runs

975 in the current experiment (about 3 degrees in the $x$ axis). Between the two processing orders,

976 the resulting $429^{\text {th }}$ volume showed negligible differences in the frontal lobe. Thus we believe the

977 order of distortion correction and motion correction was not critical. For the anatomical data,

978 the magnetic field inhomogeneity was corrected by dividing the T1 images with the PD images.

979 The anatomical data was then spatially normalized into the Talairach space.

980 To ensure that all functional runs of each participant ( 6 runs of the main experiment, 2

981 functional localizer runs, from two scanning sessions, distortion-corrected) were aligned well

982 with each other, we used a manual across-run alignment procedure, with careful visual

983 inspections and multiple iterations/checks. The first volume of the main-task run was aligned to

984 the anatomy, and then saved as an anatomical file in native space, keeping the $12 *$ weighted

985 contrast the same as the original functional run. This served as a "dummy" anatomical run for

986 the subsequent alignment procedure. Then the first volumes of all the functional runs (including

987 the first run itself) were aligned to this dummy anatomical run, and normalized into the

988 Talairach space (with the position information and transformation matrices for the T1 weighted

989 anatomical run). The first volumes of the 8 runs aligned in the Talairach space were then screen-

990 captured and saved as different layers in Photoshop (version CS6, Adobe, United States), toggled

991 on and off, and made into GIF animations to check alignment qualities across runs. Adjustments

992 were made for imperfect aligned runs, followed by the same checking procedure, until there

993 were not any big shifts/translations across runs at the whole-brain level. This procedure allowed 
us to visually spot tiny alignment imperfections across runs with the same T2* weighted modality. It is much easier than spotting misalignments across $\mathrm{T} 2 *$ weighted and T1 weighted image modalities in the conventional alignment procedure, where the two images look very different across modalities. The quality of the distortion correction was also checked when aligning the functional run 1 to the anatomy, where a good distortion correction resulted in a good shape correspondence between the T2* weighted and T1 weighted data. In some cases, when the participants moved their head between the distortion correction run and the functional task run, despite our repetitive instructions, the distortion correction quality was affected. In those cases, we strived for aligning the occipital and temporal lobes.

After alignment across runs, the functional data of the main experiment were then spatially smoothed with $6 \mathrm{~mm}$ FWHM for univariate analysis (comparable to the RSA searchlight radius), $3 \mathrm{~mm}$ FWHM for task residual functional analysis. The unsmoothed data were used for the representational similarity analysis, and univariate analysis of 10 categories in RSA searchlight clusters. The static functional localizer data was smoothed at $6 \mathrm{~mm}$ FWHM for group randomeffect GLM, $3 \mathrm{~mm}$ for individual participants, and not smoothed for univariate analysis in RSA searchlight clusters.

All results in this study were computed in the volume space. For visualizing group-level results, cortex-based alignments (CBA) were performed to alleviate the high inter-individual anatomical variability. For each participant, the gray-white-matter boundary of the anatomical images in Talairach space underwent automatic segmentation, and careful manual correction slice-byslice. Then a mesh with a high number of vertices was created for the white-gray-matter boundary of each hemisphere (number of vertices ranging from $370 \mathrm{k}$ to $434 \mathrm{k}$ ), inflated into a sphere, corrected for distortions across vertices, and mapped to a high-resolution standard sphere with down sampling (number of vertices=163842). The curvature patterns of the original mesh were also smoothed and mapped to the standard sphere. The group-level cortex-based alignment was separately performed for the left and right hemispheres. For each hemisphere, the curvature patterns of all 10 participants' meshes were aligned into a dynamic group average. After aligning, the average left and right hemispheres of the whole group were created. This resulting group-average brain surface retained many anatomical landmarks, and was more detailed than the ones usually created with 3T data. However, note that it was a bit smaller than the actual brain, and was only meant to provide visual landmarks to localize the clusters.

\section{Univariate analysis}

1027

To compare the univariate activation to the literature, three different general linear models (GLMs, serial correlation correction: AR(2), data \%-normalized before performing the GLM) were applied to the datasets smoothed $6 \mathrm{~mm}$ FWHM, with the following predictors: 1) 10 action categories; 2) the behavioral ratings of implied motion; 3 ) the behavioral ratings of valence. For 2 ) and 3), the presentation of all stimuli were defined as a single main predictor, the ratings for 40 stimuli were z-scored within each participant, and served as a parametric weight factor to each corresponding stimulus. The parametric modulation effects were subsequently computed for 2) and 3). For all the GLM models above, the time courses were \%-transformed, the main 
1034

1035

1036

1037

1038

1039

1040

1041

1042

1043

1044

1045

1046

1047

1048

1049

1050

1051

1052

1053

1054

1055

1056

1057

1058

1059

1060

1061

1062

1063

1064

1065

1066

1067

1068

1069

1070

1071

predictors were convolved with a two-gamma hemodynamic response function, and the 6 parameters of participant's head motion were z-scored and entered as confounding factors. The catch trials were marked as a separate condition, and their parametric estimates (beta values) were not used in subsequent analyses.

The group random-effect GLM analysis was performed for each predictor set. For the 10category predictor set, apart from the contrasts between action categories, a whole-brain ANOVA was performed, with a 10-level factor 'action categories'. Cluster size thresholds for all group-level maps in this study were estimated using Monte-Carlo simulation (alpha level=0.05, numbers of iterations $=5000$, initial $p<0.005$ for univariate and RSA searchlight analyses; initial $p<0.001$ for task-residual functional connectivity), with the BrainVoyager plugin Cluster-Level Statistical Threshold Estimator (https://support.brainvoyager.com/brainvoyager/functionalanalysis-statistics/46-tresholding-multiple-comparisons-problem/226-plugin-help-clusterthresholding), masked with the common functional data coverage across 10 participants. This mask was created from the averaged functional images across participants, covering 830178 functional voxels.

The GLM of the static functional localizer (without smoothing, and smoothed $3 \mathrm{~mm}$ FWHM) was performed for each individual participant. Since we do not assume that the bodies should only be processed in category-specific areas, the static functional localizer data were used as an indicator for inter-individual variability, and an indicator for the neural processes in some RSA searchlight clusters.

\section{Representational similarity analysis for fMRI data}

For RSA analyses, the GLM model with 40 stimuli was fitted to each individual participants' unsmoothed data, resulting in $40 \mathrm{t}$-maps, one per stimulus. The neural RDMs across the 40 stimuli were computed with Pearson's correlation distance Searchlight spheres were constructed for each voxel, with a radius of 5 voxels ( 515 voxels within the sphere, $889.92 \mathrm{~mm}^{3}$ ), comparable to the value for univariate smoothing. Spheres containing more than 172 non-zero voxels were included in the analysis). The Spearman correlation values between the neural RDM and the model RDM was Fisher-Z transformed, and wrote back to the sphere center voxel. The group-level significance of the correlation was evaluated by a one-sample t-test against zero (2tailed).

For the clusters found by the searchlight RSA and subsequent whole-brain analyses, the univariate percent signal changes for each of the 10 action categories were extracted (without smoothing), and were compared to the baseline (one-sample t test against 0 ) in SPSS.

\section{RSA regression with low/mid-level features}

For each of the stimuli image, we extracted the body joint locations by the OpenPose library (Cao et al., 2017) (https://github.com/CMU-Perceptual-Computing-Lab/openpose). The output was the $x, y$ values and a confidence score for the estimation, for 18 body joints (See Figure 4A). The joint locations were imported in Adobe Illustrator CS6, overlaid on the stimulus image, and 
manually adjusted with visual estimation. The correspondence of the joints and their location are as follows: joint 1 (head)-nose tip; joint 2 (neck)-the jugular notch of the sternum; joint 3 \& 6 (shoulders)-humerus; joint $4 \& 7$ (elbows)-the connecting point of the upper/lower arms; joint 5 $\& 8$ (hands)-the wrist end of the radial bone; joint $9 \& 12$ (waist)-the widest points of the femur; joint $10 \& 13$ (knees)-on the patella; joint $11 \& 14$ (feet)-the ankle end of the tibia; joint $15 \& 16$ (eyes); joint $17 \& 18$ (ears). Since the eyes were not present on the stimuli images (facial

1078 features blurred), and the ears were often occluded by the hair or by the head, these joints were 1079 visually estimated with linear perspective in mind.

1080 We constructed low-level visual feature RDMs, for raw pixel values (as vectors), and raw coordinates of the 14 joints (excluding joints for ears and eyes), although the resulting RDMs were extremely similar to the ones including these 4 joints (RDM similarity rho $=0.976,0.98$ for stimuli set $A$ and $B$ ).

The mid-level visual feature RDMs included head, shoulder, waist orientations, as well as hands/feet-to-head distances. The head orientation was individually computed by the distance between ears, normalized (divided) by the ear distance of the standing still condition, capped at 1 (1: facing the viewer; smaller than 1: facing left- or right-ward). The shortest hand-to-head distance may reflect whether the hand enters the head's peripersonal space of the actor; the average feet-to-head distance may reflect the relaxation of the torso and legs of that actor. RDMs and the higher-level stimuli attribute RDMs (implied motion, valence, actor identity) were squared for each element (to satisfy the linearity for squared Euclidean distance values), zscored across elements, then put together in the same linear regression model as predictors. Each element of the neural RDM was also correspondingly squared. A set of beta values for these predictors were obtained for each participant, and their group-level significance was examined by one-sample t-test against zero (2-tailed), and FDR-corrected at $\mathrm{q}<0.05$ (Storey, 2002) across all predictors for each searchlight cluster.

\section{Task residual functional connectivity analysis}

1100 We smoothed the task data at $3 \mathrm{~mm}$ FWHM, regressed out the task-related activity by 1101 deconvolution analysis (5 stick predictors per stimulus, covering the evolvement of the BOLD 1102 shape per trial). For each seed region, the residual time course was extracted and averaged 1103 across voxels, and correlated with the residual time courses of all voxels in each run, resulting in 1104 one R map per run. The connectivity pattern across the runs were stable for all participants. The

1105 R maps were Fisher's Z-transformed, averaged across runs per participant, and put in a one1106 sample t-test against 0 ( 2 tailed). The resulting group-level maps were thresholded at $p<0.001$ 1107 for cluster size correction. 
For the 10-action-category areas, the inter-area functional connectivity was also examined, by correlating the seed ROI time courses per run, then again with the same group-analysis procedure (Z-transform, averaging, t test), with FDR correction.

1111 Hierarchical clustering of RDMs across functional connectivity clusters

1112 We computed neural RDMs for all group-level clusters obtained from the task-residual

1113 functional connectivity analysis (51 ROIs for the perceived action FC network, 76 ROIs for the 1114 perceived emotion FC network), and computed second-level RDMs (Spearman correlation 1115 distance, Figure 6F) from them within each participant. For the two FC networks, the second1116 level RDM were averaged (with Z-transform) across participants (Figure 6A, B, right panels), and 1117 served as inputs for hierarchical clustering (MATLAB function: linkage; method: average. Plot 1118 function: dendrogram; clustering threshold for the most similar areas to the seed region: 0.8). 1119 We also examined the univariate activation for 10 action categories, and plotted histograms.

\section{References}

Amodio, D.M., and Frith, C.D. (2006). Meeting of minds: the medial frontal cortex and social cognition. Nature Reviews Neuroscience 7, 268-277.

Barrett, L.F., Adolphs, R., Marsella, S., Martinez, A.M., and Pollak, S.D. (2019). Emotional

1133 Binder, J.R., Desai, R.H., Graves, W.W., and Conant, L.L. (2009). Where is the semantic system? A 1134 critical review and meta-analysis of 120 functional neuroimaging studies. Cereb Cortex 19, 1135 2767-2796.

1136 Boleda, G. (2020). Distributional Semantics and Linguistic Theory. Annual Review of Linguistics 6, 1137 213-234.

1138 Bracci, S., and Op de Beeck, H. (2016). Dissociations and Associations between Shape and 1139 Category Representations in the Two Visual Pathways. J. Neurosci. 36, 432-444.

1140 Buckner, R.L., Andrews-Hanna, J.R., and Schacter, D.L. (2008). The Brain's Default Network. 1141 Annals of the New York Academy of Sciences 1124, 1-38. 
1142 Bufacchi, R.J., Liang, M., Griffin, L.D., and lannetti, G.D. (2016). A geometric model of defensive 1143 peripersonal space. Journal of Neurophysiology 115, 218-225.

1144 Bullmore, E., and Sporns, O. (2009). Complex brain networks: graph theoretical analysis of 1145 structural and functional systems. Nature Reviews Neuroscience 10, 186-198.

1146 Cao, Z., Simon, T., Wei, S.-E., and Sheikh, Y. (2017). Realtime Multi-person 2D Pose Estimation 1147 Using Part Affinity Fields. (IEEE), p.

1148 Cao, Z., Hidalgo, G., Simon, T., Wei, S.-E., and Sheikh, Y. (2019). OpenPose: Realtime Multi1149 Person 2D Pose Estimation using Part Affinity Fields. ArXiv:1812.08008 [Cs].

1150 Caspers, S., Zilles, K., Laird, A.R., and Eickhoff, S.B. (2010). ALE meta-analysis of action 1151 observation and imitation in the human brain. Neuroimage 50, 1148-1167.

1152 Charest, I., Kievit, R.A., Schmitz, T.W., Deca, D., and Kriegeskorte, N. (2014). Unique semantic 1153 space in the brain of each beholder predicts perceived similarity. PNAS 111, 14565-14570.

1154 Chikazoe, J., Lee, D.H., Kriegeskorte, N., and Anderson, A.K. (2014). Population coding of affect 1155 across stimuli, modalities and individuals. Nat Neurosci 17, 1114-1122.

1156 Connolly, A.C., Guntupalli, J.S., Gors, J., Hanke, M., Halchenko, Y.O., Wu, Y.-C., Abdi, H., and 1157 Haxby, J.V. (2012). The Representation of Biological Classes in the Human Brain. Journal of 1158 Neuroscience 32, 2608-2618.

1159 Cowen, A.S., and Keltner, D. (2017). Self-report captures 27 distinct categories of emotion 1160 bridged by continuous gradients. PNAS 114, E7900-E7909.

1161 Cowen, A.S., and Keltner, D. (2020). What the face displays: Mapping 28 emotions conveyed by 1162 naturalistic expression. American Psychologist 75, 349-364.

1163 Cowen, A.S., and Keltner, D. (2021). Semantic Space Theory: A Computational Approach to 1164 Emotion. Trends in Cognitive Sciences 25, 124-136.

1165 Cowen, A.S., Elfenbein, H.A., Laukka, P., and Keltner, D. (2019). Mapping 24 emotions conveyed 1166 by brief human vocalization. American Psychologist 74, 698-712.

1167 DiCarlo, J.J., and Cox, D.D. (2007). Untangling invariant object recognition. Trends in Cognitive 1168 Sciences 11, 333-341.

1169 DiCarlo, J.J., Zoccolan, D., and Rust, N.C. (2012). How Does the Brain Solve Visual Object 1170 Recognition? Neuron 73, 415-434.

1171 Dricu, M., and Frühholz, S. (2016). Perceiving emotional expressions in others: Activation 1172 likelihood estimation meta-analyses of explicit evaluation, passive perception and incidental 1173 perception of emotions. Neuroscience \& Biobehavioral Reviews 71, 810-828.

1174 Ekman, P. (1999). Basic emotions. Handbook of Cognition and Emotion 98, 16. 
1175 Freedman, D.J., Riesenhuber, M., Poggio, T., and Miller, E.K. (2001). Categorical Representation 1176 of Visual Stimuli in the Primate Prefrontal Cortex. Science 291, 312-316.

1177 de Gelder, B. (2006). Towards the neurobiology of emotional body language. Nature Reviews 1178 Neuroscience 7, 242-249.

1179 de Gelder, B., and Van den Stock, J. (2011). The Bodily Expressive Action Stimulus Test (BEAST). 1180 Construction and Validation of a Stimulus Basis for Measuring Perception of Whole Body 1181 Expression of Emotions. Front Psychol 2, 181-181.

1182 de Gelder, B., Snyder, J., Greve, D., Gerard, G., and Hadjikhani, N. (2004). Fear fosters flight: a 1183 mechanism for fear contagion when perceiving emotion expressed by a whole body. Proc Natl 1184 Acad Sci U S A 101, 16701-16706.

1185 Glasser, M.F., Coalson, T.S., Robinson, E.C., Hacker, C.D., Harwell, J., Yacoub, E., Ugurbil, K., 1186 Andersson, J., Beckmann, C.F., Jenkinson, M., et al. (2016). A multi-modal parcellation of human 1187 cerebral cortex. Nature 536, 171-178.

1188 Goeleven, E., De Raedt, R., Leyman, L., and Verschuere, B. (2008). The Karolinska Directed 1189 Emotional Faces: A validation study. Cognition \& Emotion 22, 1094-1118.

1190 Grafton, S.T., and Hamilton, A.F. de C. (2007). Evidence for a distributed hierarchy of action 1191 representation in the brain. Hum Mov Sci 26, 590-616.

1192 Hartley, C.A., and Poeppel, D. (2020). Beyond the Stimulus: A Neurohumanities Approach to 1193 Language, Music, and Emotion. Neuron 108, 597-599.

1194 Haxby, J.V., Guntupalli, J.S., Connolly, A.C., Halchenko, Y.O., Conroy, B.R., Gobbini, M.I., Hanke, 1195 M., and Ramadge, P.J. (2011). A Common, High-Dimensional Model of the Representational 1196 Space in Human Ventral Temporal Cortex. Neuron 72, 404-416.

1197 Hebart, M.N., Dickter, A.H., Kidder, A., Kwok, W.Y., Corriveau, A., Wicklin, C.V., and Baker, C.I. 1198 (2019). THINGS: A database of 1,854 object concepts and more than 26,000 naturalistic object 1199 images. PLOS ONE 14, e0223792.

1200 Hein, G., and Knight, R.T. (2008). Superior Temporal Sulcus-It's My Area: Or Is It? Journal of 1201 Cognitive Neuroscience 20, 2125-2136.

1202 Hoemann, K., Wu, R., LoBue, V., Oakes, L.M., Xu, F., and Barrett, L.F. (2020). Developing an 1203 Understanding of Emotion Categories: Lessons from Objects. Trends in Cognitive Sciences 24, $120439-51$.

$1205 \mathrm{Ju}, \mathrm{H}$. , and Bassett, D.S. (2020). Dynamic representations in networked neural systems. Nature 1206 Neuroscience 23, 908-917.

1207 Kanai, R., and Rees, G. (2011). The structural basis of inter-individual differences in human 1208 behaviour and cognition. Nature Reviews Neuroscience 12, 231-242. 
King, M.L., Groen, I.I.A., Steel, A., Kravitz, D.J., and Baker, C.I. (2019). Similarity judgments and cortical visual responses reflect different properties of object and scene categories in naturalistic images. Neurolmage 197, 368-382.

1212 Kober, H., Barrett, L.F., Joseph, J., Bliss-Moreau, E., Lindquist, K., and Wager, T.D. (2008). 1213 Functional grouping and cortical-subcortical interactions in emotion: A meta-analysis of 1214 neuroimaging studies. Neurolmage 42, 998-1031.

Kret, M.E., and de Gelder, B. (2010). Social context influences recognition of bodily expressions. perceiving threat from dynamic faces and bodies. An fMRI study. Neurolmage 54, 1755-1762.

1219 Kriegeskorte, N., Mur, M., Ruff, D.A., Kiani, R., Bodurka, J., Esteky, H., Tanaka, K., and Bandettini, 1220 P.A. (2008). Matching categorical object representations in inferior temporal cortex of man and 1221 monkey. Neuron 60, 1126-1141.

1222 de Lange, F.P., Spronk, M., Willems, R.M., Toni, I., and Bekkering, H. (2008). Complementary 1223 Systems for Understanding Action Intentions. Current Biology 18, 454-457.

1224 Langner, O., Dotsch, R., Bijlstra, G., Wigboldus, D.H.J., Hawk, S.T., and van Knippenberg, A.

1225 (2010). Presentation and validation of the Radboud Faces Database. Cognition \& Emotion 24, 1226 1377-1388.

1227 LeDoux, J.E., and Hofmann, S.G. (2018). The subjective experience of emotion: a fearful view. 1228 Current Opinion in Behavioral Sciences 19, 67-72.

1229 Lindquist, K.A., Wager, T.D., Kober, H., Bliss-Moreau, E., and Barrett, L.F. (2012). The brain basis 1230 of emotion: a meta-analytic review. Behav Brain Sci 35, 121-143.

1231 Lindquist, K.A., Gendron, M., Feldman Barrett, L., and Dickerson, B.C. (2014). Emotion

1232 perception, but not affect perception, is impaired with semantic memory loss. Emotion 14, 3751233387.

1234 Lundqvist, D., Flykt, A., and Öhman, A. (1998). Karolinska Directed Emotional Faces.

1235 Mansouri, F.A., Freedman, D.J., and Buckley, M.J. (2020). Emergence of abstract rules in the 1236 primate brain. Nature Reviews Neuroscience 21, 595-610.

1237 Masson, H.L., and Isik, L. (2021). Functional selectivity for naturalistic social interaction 1238 perception in the human superior temporal sulcus. BioRxiv 2021.03.26.437258.

1239 Mikolov, T., Chen, K., Corrado, G., and Dean, J. (2013). Efficient Estimation of Word 1240 Representations in Vector Space. ArXiv:1301.3781 [Cs].

1241 Moeller, S., Yacoub, E., Olman, C.A., Auerbach, E., Strupp, J., Harel, N., and Uğurbil, K. (2010). 1242 Multiband multislice GE-EPI at 7 tesla, with 16-fold acceleration using partial parallel imaging 
1243 with application to high spatial and temporal whole-brain fMRI. Magn Reson Med 63, 1144-

12441153.

1245 Molenberghs, P., Cunnington, R., and Mattingley, J.B. (2012). Brain regions with mirror 1246 properties: A meta-analysis of 125 human $\mathrm{fMRI}$ studies. Neuroscience \& Biobehavioral Reviews $124736,341-349$.

1248 Mur, M., Meys, M., Bodurka, J., Goebel, R., Bandettini, P.A., and Kriegeskorte, N. (2013). Human 1249 Object-Similarity Judgments Reflect and Transcend the Primate-IT Object Representation. Front. 1250 Psychol. 4.

1251 Nili, H., Wingfield, C., Walther, A., Su, L., Marslen-Wilson, W., and Kriegeskorte, N. (2014). A 1252 toolbox for representational similarity analysis. PLoS Comput Biol 10, e1003553-e1003553.

1253 Peelen, M.V., Atkinson, A.P., and Vuilleumier, P. (2010). Supramodal representations of 1254 perceived emotions in the human brain. J Neurosci 30, 10127-10134.

1255 Peelen, M.V., He, C., Han, Z., Caramazza, A., and Bi, Y. (2014). Nonvisual and Visual Object Shape 1256 Representations in Occipitotemporal Cortex: Evidence from Congenitally Blind and Sighted 1257 Adults. Journal of Neuroscience 34, 163-170.

1258 Peirce, J.W. (2007). PsychoPy--Psychophysics software in Python. J Neurosci Methods 162, 8-13.

1259 Pelli, D.G. (1997). The VideoToolbox software for visual psychophysics: transforming numbers 1260 into movies. Spatial Vision 10, 437-442.

1261 Pilehvar, M.T., and Collier, N. (2016). De-Conflated Semantic Representations. (Association for 1262 Computational Linguistics), $\mathrm{p}$.

1263 Righart, R., and de Gelder, B. (2006). Context Influences Early Perceptual Analysis of Faces -An 1264 Electrophysiological Study. Cerebral Cortex 16, 1249-1257.

1265 Rizzolatti, G., Cattaneo, L., Fabbri-Destro, M., and Rozzi, S. (2014). Cortical Mechanisms 1266 Underlying the Organization of Goal-Directed Actions and Mirror Neuron-Based Action 1267 Understanding. Physiological Reviews 94, 655-706.

1268 Saxe, R., Moran, J.M., Scholz, J., and Gabrieli, J. (2006). Overlapping and non-overlapping brain 1269 regions for theory of mind and self reflection in individual subjects. Soc Cogn Affect Neurosci 1, $1270 \quad 229-234$.

1271 Seger, C.A. (2008). How do the basal ganglia contribute to categorization? Their roles in 1272 generalization, response selection, and learning via feedback. Neurosci Biobehav Rev 32, 2651273278.

1274 Seger, C.A., and Miller, E.K. (2010). Category learning in the brain. Annu Rev Neurosci 33, 2031275219.

1276 Seghier, M.L., and Price, C.J. (2018). Interpreting and Utilising Intersubject Variability in Brain 1277 Function. Trends in Cognitive Sciences 22, 517-530. 
1278 Sinke, C.B.A., Sorger, B., Goebel, R., and de Gelder, B. (2010). Tease or threat? Judging social

1279 interactions from bodily expressions. Neurolmage 49, 1717-1727.

1280 Skerry, A.E., and Saxe, R. (2015). Neural Representations of Emotion Are Organized around 1281 Abstract Event Features. Current Biology 25, 1945-1954.

1282 Smith, P.L., and Little, D.R. (2018). Small is beautiful: In defense of the small-N design. Psychon 1283 Bull Rev 25, 2083-2101.

1284 Stienen, B.M.C., and de Gelder, B. (2011). Fear detection and visual awareness in perceiving 1285 bodily expressions. Emotion 11, 1182-1189.

1286 Stolier, R.M., and Freeman, J.B. (2016). Neural pattern similarity reveals the inherent 1287 intersection of social categories. Nature Neuroscience 19, 795-797.

1288 Storey, J.D. (2002). A direct approach to false discovery rates. Journal of the Royal Statistical 1289 Society: Series B (Statistical Methodology) 64, 479-498.

1290 Tucciarelli, R., Wurm, M., Baccolo, E., and Lingnau, A. (2019). The representational space of 1291 observed actions. ELife 8, e47686.

1292 Vaessen, M., Heijden, K.V. der, and Gelder, B. de (2019a). Decoding of emotion expression in the 1293 face, body and voice reveals sensory modality specific representations. BioRxiv 869578.

1294 Vaessen, M.J., Abassi, E., Mancini, M., Camurri, A., and de Gelder, B. (2019b). Computational 1295 Feature Analysis of Body Movements Reveals Hierarchical Brain Organization. Cerebral Cortex $129629,3551-3560$.

1297 Van den Stock, J., Hortensius, R., Sinke, C., Goebel, R., and de Gelder, B. (2015). Personality traits 1298 predict brain activation and connectivity when witnessing a violent conflict. Scientific Reports 5.

1299 Van Overwalle, F., and Baetens, K. (2009). Understanding others' actions and goals by mirror 1300 and mentalizing systems: A meta-analysis. Neurolmage 48, 564-584.

1301 Yeo, B.T., Krienen, F.M., Sepulcre, J., Sabuncu, M.R., Lashkari, D., Hollinshead, M., Roffman, J.L., 1302 Smoller, J.W., Zöllei, L., Polimeni, J.R., et al. (2011). The organization of the human cerebral 1303 cortex estimated by intrinsic functional connectivity. Journal of Neurophysiology 106, 112513041165.

1305 Zhang, Y., Han, K., Worth, R., and Liu, Z. (2020). Connecting concepts in the brain by mapping 1306 cortical representations of semantic relations. Nature Communications 11, 1877. 